\title{
EIN UNBEKANNTES KRIEGSBILD VON OTTO DIX - ZUR FRAGE DER ABFOLGE SEINER KRIEGSARBEITEN 1915-1918
}

\author{
von DIETRICH SCHUBERT
}

für O.K. Werckmeister

»Der Maler ist das Auge der Welt, der Maler lehrt die Menschen sehen...«(Dix)

\begin{abstract}
Der 100. Geburtstag des Realisten Otto Dix 1991 hatte zwar eine Reihe von Ausstellungen mit entsprechenden Katalogen gebracht (Albstadt, Dresden, Gera, Stuttgart-Berlin-London, Friedrichshafen), was aber die wichtigste Facette seines Werkes - die verschiedenen Darstellungen des 1. Weltkriegs und seiner Opfer - betriff, so gibt es nur wenige neue Beiträge bzw. Erkenntnisse wie die Publikation der Geraer Feldpostkarten 1991 durch Ulrike Rüdiger. - Wegen häufig ungenauer Datierungen der Zeichnungen und Gouachen von Dix aus dem Krieg 1914-18 und weil diese keineswegs zur Gänze gesammelt oder publiziert sind - das Werkverzeichnis der Gouachen von Suse Pfäfle ist unvollständig - bemüht sich der Autor gegenüber der älteren Literatur um Kriterien der Abfolge der Kriegszeichnungen von Dix; Anlaß ist die meisterhafte Gouache von 1917, die hier erstmals veröffentlicht wird. - Es zeigt sich beim heutigen Überblick über den Stand der Forschung zu Dix, daß eine konzentrierte Publikation der vielen hundert Zeichnungen aus den Kriegsjahren September 1915 bis November 1918 ein wirkliches Desiderat ist. (Der Beitrag wurde bereits 1993 verfaßt.)
\end{abstract}

I.

Immer wieder stößt die Forschung zu den von Dix während des Krieges 1915-1918 geschaffenen Zeichnungen und Gouachen verschiedener FormModi, die ein Spektrum an Gestaltungsweisen von naturalistisch über realistisch, kubistisch und kubofuturistisch $\mathrm{zu}$ weitgehender Abstraktion (bis $\mathrm{zu}$ den schwarzen Tusch-Zeichnungen um 1918/19) aufweisen, auf unklare Fragen, an Hürden, Leseschwierigkeiten, ja weiße Flecken und Fallen. Datierungsprobleme für diese Kriegswerke sind dann die Folge.

Wenn in dem aufwendig gedruckten Werkverzeichnis der Aquarelle und Gouachen von Suse Pfäffle von 1991 z.B. noch zu lesen ist: "Das Blatt

\footnotetext{
1 Suse Pfäffle, Otto Dix - Werkverzeichnis der Aquarelle und Gouachen, Stuttgart 1991 (zur großen Retrospektive in Stuttgart/Berlin eilig fertiggestellt, aber unvollständig), S. 256 und 269.

2. Vergleiche zur Biographie aus der großen Zahl der in den letzten Jahren publizierten Kataloge etwa Rainer Beck/O.
}

wird zwischen 1915 und 1917 entstanden sein«, so genügt solche Arbeit keineswegs kunstwissenschaftlichen Kriterien und erweist letztlich ein mangelhaftes Formsehen der Autorin. ${ }^{1}$

Selbst die verschiedenen Ausstellungen der Jahre 1991/92 in Stuttgart, Berlin, Friedrichshafen, Gera, Albstadt, Dresden und London haben für das signifikante Kriegs-Itinerar von Dix, das zwar inzwischen in den Stationen relativ deutlich ist, aber für die Abfolge der Zeichnungen und Gouachen noch Unklarheiten aufweist - ich denke hier besonders an den Aufenthalt an der Ostfront bei Lagoerde und Gorodniki im Spätherbst 1917, d.h. Oktober bis Dezember 1917 - keine neuen Fakten gebracht. ${ }^{2}$

Grundlagen sind insbesondere der Militärpaß im Germanischen Nationalmuseum Nürnberg und

Conzelmann (Hrsg.), OTTO DIX - Kat. der Ausstellung der Villa Stuck, München 1985; - Dietrich Schubert: OTTO DIX (1980), 3., verbesserte Aufl. Reinbek 1991; - Andrea Hollmann, in: Otto Dix - zum 100. Geburtstag, hrsg. v. Johann K.Schmidt/Wulf Herzogenrath, Stuttgart/Berlin 1991, S. 11-26. 
die in Gera aufbewahrten 46 gezeichneten Feldpostkarten im Format $14 \times 9 \mathrm{~cm}$ plus 7 Fotografien an die Freundin Helene Jakob/Dresden, die von mir bereits 1978 für die Rowohlt-Monographie konsultiert worden waren und die inzwischen in einer mustergültigen Publikation durch Ulrike Rüdiger zugänglich sind. ${ }^{3}$

Damit ist das so wichtige Kriegs-Itinerar zwar noch nicht komplett, aber für große Teile ab dem Kriegsdienst von Dix seit dem 21.9.1915 in der Champagne vor Reims konstituiert. ${ }^{4}$ Dies ist von Bedeutung, weil es im 1. Weltkrieg keinen anderen Maler oder Bildhauer gegeben hat, der das Kriegsgeschehen und Menschenmorden derart lange und intensiv an vorderster Front als MG-Schütze erlebt und derartig breit und differenziert dargestellt hat (und noch dazu auch in verschiedenen Phasen seines späteren Schaffens) wie der Thüringer Arbeitersohn Otto Dix, - auch nicht Albert Weisgerber (der im Mai 1915 fiel), auch nicht Beckmann (der im Juni 1915 mit Nervenzusammenbruch ausschied), ${ }^{5}$ - auch nicht der schwerverletzte Kokoschka, nicht Boccioni, nicht Franz Mare (der 1916 bei einem Transport umkam), nicht Wilhelm Gerstel, nicht Lehmbruck, nicht Waldemar Rösler (der die gleichen Erlebnisse wie Dix an der Westfront bei Ypern bis 1916 hatte, dann Selbstmord beging), nicht Paul Klee, ${ }^{6}$ nicht der Bildhauer Christoph Voll,

3 Der Militärpaß von Dix hat sich - im Gegensatz zu denen anderer Künstler im 1. Weltkrieg wie Christoph Voll - im DixArchiv im GNM Nürnberg erhalten, p. 10/11 führt die Teilnahme an Schlachten auf, die Auszeichnungen und Beförderung zum Unteroffizier am 1.11.1915; es folgen Krankheiten und Verwundungen, ferner die Kommandos wie der Kursus zur Flieger-Abwehr mit schwerem M.G. im August 1917 in Gent, ferner Orden, Ehrenzeichen, Beförderungen, Führung, Strafen, Versetzungen usf. Für die Jahre nach 1916 wurden dann von den Vorgesetzten keine handschriftlichen Einträge mehr gemacht, sondern Vordrucke benutzt (p. 16), die jedoch mißverständlich bleiben, weil sie per Hand korrigiert wurden. 4 Siehe Dietrich Schubert, DIX (wie Anm. 2), 3. Aufl. 1991, 4. A. 1996 , S. $22-28$ (in der 1. Aufl. noch fehlerhaft). - Ulrike Rüdiger, Grüsse aus dem Krieg - die Feldpostkarten aus der DixSammlung Gera, Gera 1991, S. 22-23 (es handelte sich ursprünglich um ca. 260 Postkarten!). - Trotz der hervorragenden Arbeit von U. Rüdiger darf man anmerken, daß ihre Abb. 8, S. 19 nicht exakt lokalisiert und nicht der Postkarte Nr. 39 von August 1917 zugeordnet wurde; die Bezeichnung des Blattes - unsere Abb. 1 - lautet links oben »DIX Knocke 17 «. Auch der Zeichenstil systematischer, kantiger Hell-DunkelMassen für die badenden Soldaten am Meer weist nach den Blättern von 1916 nun deutlich den kubo-futuristischen Modus auf, einen Zeichenstil, der stärker systematisiert, Rundes und Kantiges klarer einsetzt als zuvor. - Wenn wir das KriegsItinerar im Sommer 1917 laut Fotokarte Gera Nr. 38 um einen Ruhe-Aufenthalt bei Brügge (und am Meer) ergänzen, ordnet sich das fragliche Blatt problemlos ein. Diese mehrwöchige Ruhe folgte auf die Schlacht bei Ypern, Juli 1917 (Mil. paß p. 16). - Vgl. von U. Rüdiger ferner den Beitrag über die Geraer Feldpostkarten in: Kat. Dix (wie Anm. 2) Stuttgart-Berlin 1991, S. 51 f., eine Kurzfassung ihres Buches. der den Krieg jahrelang wie Dix als MG-Truppführer und Unteroffizier vom April 1915 bis November 1918 überlebte, aber nicht gestaltete, nicht zeichnete, keine Gouachen schuf, den Krieg nicht als tiefes existenzielles Erlebnis nutzte wie Beckmann, den Krieg also auch nicht deutete, auch nicht nach 1919 wie Dix in seinen Verarbeitungen um 1922/24 und in der Mappe von 70 Radierungen 1924, "DER KRIEG «, wohl die bedeutendste Darstellung. ${ }^{7}$

Bis auf die unklare Zuordnung der "Knocke«Zeichnung (Abb. 1) zum Ruhe-Aufenthalt bei Brügge im August 1917 und die Frage der Verbindlichkeit der Vordrucke im Dix-Militärpaß p. 16 zu Dix im besonderen hat die Studie von U. Rüdiger ein solides Fundament gelegt für die weitere Differenzierung unserer Kenntnisse von Dix im 1. Weltkrieg.

Denn häufig begannen die Probleme bereits mit dem Lesen der Titel französischer Ortsnamen auf den Dix-Blättern: "Thugny“ (nicht Thumy) oder Bétheniville (Abb. 2) - jüngst auf einem Blatt einer Lempertz-Auktion 1990 nicht exakt identifiziert. ${ }^{8}$ Der kleine Ort liegt an der Suippe, bei Pont Faverger (östlich Reims), in der Nähe von Aubérive, das auf zahlreichen Blättern und Postkarten (in Gera) von Februar-März 1916 als Konnotation erscheint. ${ }^{9}$

Der Blick auf eine Karte von Aisne und Suippe bei Reims etwa aus den »Sanitätsberichten über das Dt. HEER im Weltkrieg 1914-18, II. Band« hätte

5 Max Beckmann, Briefe im Kriege (Berlin 1916), 3. Aufl. München 1984; vgl. Dietrich Schubert, Max Beckmann - Auferstehung und Erscheinung der Toten, Worms 1985.

$6 \mathrm{Zu}$ Waldemar Rösler vgl. Katalog der Ausstellung RÖSLER, hrsg. von Werner Timm, Ostdt. Galerie Regensburg 1982, S. $12 \mathrm{f}$. - Walter S. Laux, Waldemar Rösler - eine Studie zur Kunst der Berliner Secession (Diss. Heidelberg 1986), Worms 1989.

Das Kriegs-Itinerar von Rösler ist in handschriftlichen Notizen des Sohnes Fritz nach den Feldpostkarten und Briefen Röslers erhalten (Nachlaß Rösler bei Louise Kröhnke-Rösler, Berlin, der ich zu Dank verpflichtet bin). - Zu Paul Klee vgl. Otto K. Werckmeister, Klee im 1. Weltkrieg, in: Versuche über Paul Klee, Frankfurt/M. 1981, S. 10-97.

$7 \mathrm{Zu}$ Christoph Voll vgl. Anne Kassay, Der Bildhauer Christoph Voll 1897-1939 (Diss. Hamburg 1986), Worms 1994.

Inzwischen wurde von mir die Militär-Dienstbescheinigung von Voll vom 9. Mai 1933 von der Reichsarchiv-Zweigstelle Dresden (wohl für seine Stellung als Akademielehrer in Karlsruhe 1953) im Archiv der Kunstakademie Karlsruhe gefunden. Voll kämpfte wie Dix an der Somme (Sept. 1916), in Flandern an der Yser (Herbst 1915), im November 1917 an der Ostfront (am Zbrucz) im Masch.Gew.Batt. XII, erhielt am 15.12.1916 das E.K. 2. Klasse, war am 30.9.1916 zum Unteroffizier befördert worden und somit wie Dix ständig kämpfender MG-Truppführer.

8 Thugny falsch gelesen im Bestandskatalog der Dix-Sammlung der Galerie der Stadt Stuttgart, hrsg. von J.K. Schmidt und Brigitte Reinhardt, Stuttgart 1989, Nr. 65. - Katalog der Lempertz-Auktion 658 vom Nov. 1990, Nr. 910 mit Abb., bezeichnet "Betheni ... 16«, also von Frühjahr 1916.

9 Vergleiche das Blatt Format 28,5 × 28,5 cm eines Eingangs zum Unterstand in Stuttgart, Galerie der Stadt, »Aubérive 16 


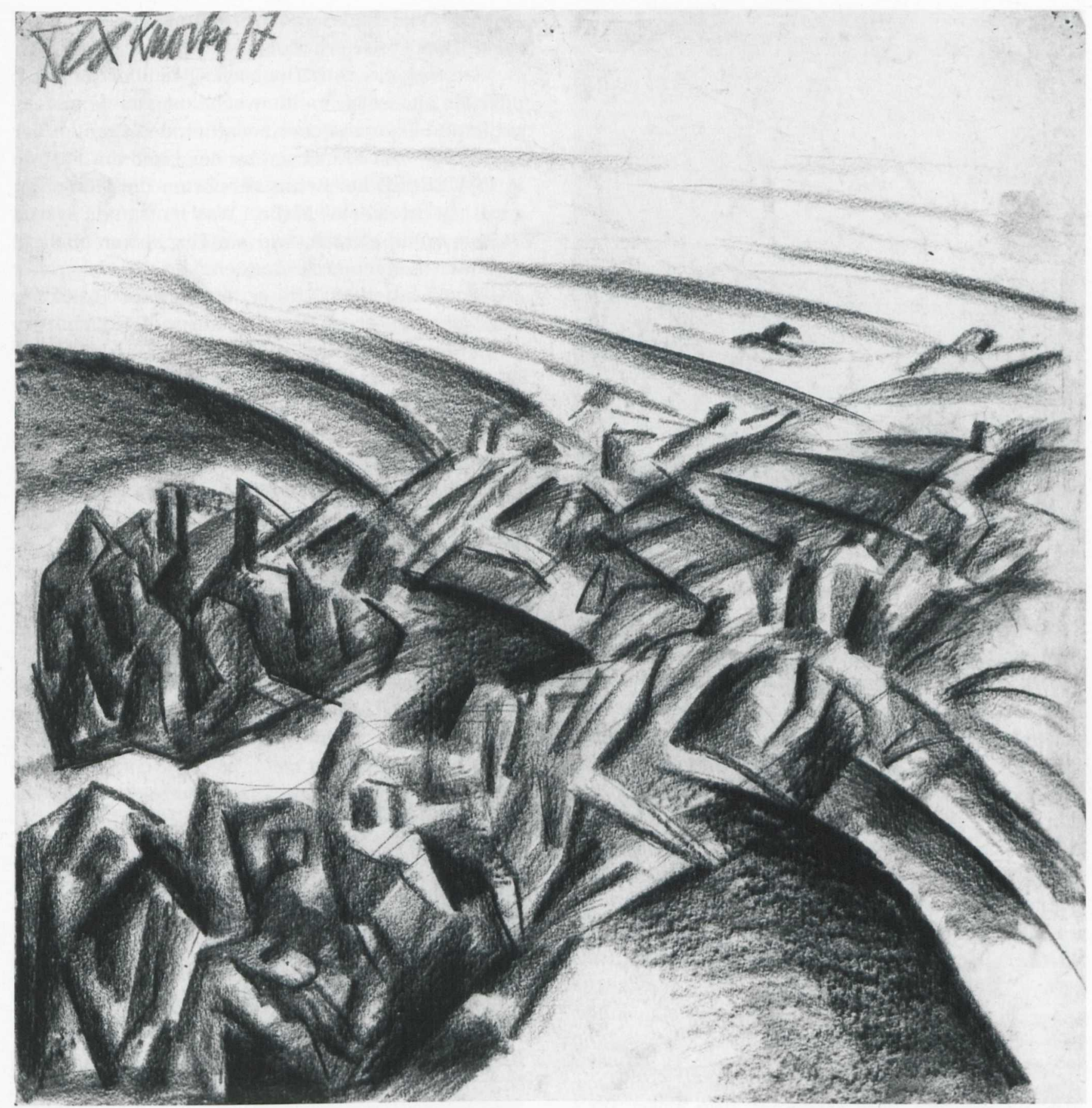

Abb. 1. Otto Dix, Badende Soldaten bei Knocke, Kreidezeichnung 1917, Privatbesitz

Klarheit über die Orte der Einsätze von Dix zwischen Thugny (bei Rethel), Pont-Faverger - Dontrien - Aubérive gebracht. Selbst O. Conzelmann, der 1983 in seinem Vorwort postulierte, gegen alle Vorurteile über Dix allein »die Wahrheit« zu bringen, datierte etliche Zeichnungen falsch: z.B. die Blätter

DIX « und ferner die Postkarten in Gera Nr. 13-15 und »Stauwerk bei A." (Gera Nr. 25) und "Straße St. Souplet-Aubérive" vom 1. Mai 1916 (Gera Nr. 29 - bei U. Rüdiger, Grüsse (wie Anm. 4), 1991).

10 Otto Conzelmann, Der andere Dix, Klett-Cotta Stuttgart 1983. - Die Aubérive-Blätter Abb. 131-133 sind auch falsch datiert. mit dem Ort "Angres" setzte er 1915 an, obgleich Angres im Artois (um Arras) liegt, wo Dix im September-Oktober 1916 kämpfte (Karte S. 168). ${ }^{10}$

Noch jüngst wurde in der Dresdner Publikation der DIX-Zeichnungen für die Ausstellungs-Beteiligung von Dix 1916. in Dresden mit elf Zeichnungen

In Angres war Dix 1915 überhaupt nicht; er rückte erst im September 1915 an die Front und war bis Juli 1916 in der Champagne; meist fehlen bei Conzelmann im Abb.-Verzeichnis die Angaben zu den Standorten der Blätter, was äußerst mißlich ist. Conzelmann arbeitete dilettantisch und oft ohne Originalkenntnis der Blätter nach den Nummern des Fotografen Kabus/Konstanz, weshalb er auch Blätter in zwei Versio- 


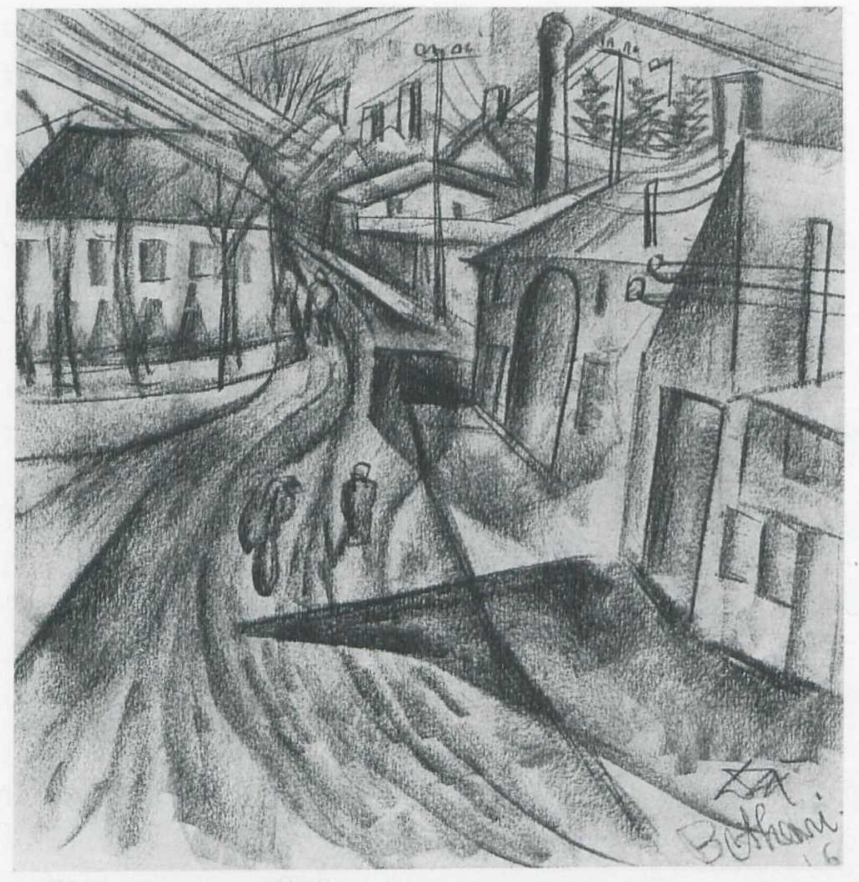

Abb. 2. Otto Dix, Dorfeingang von Bétheniville, schwarze Kreide, Frühjahr 1916, Privatbesitz

in der Galerie Ernst Arnold/Schloßstrasse die Nr. 243 falsch als "Vontrieu« gelesen; es handelt sich aber um eine Skizze (im Normformat 28/29 × 28/29 $\mathrm{cm})$, die das zerstörte Dorf Dontrien, zwischen Bétheniville und Aubérive, zeigt. ${ }^{11}$ Bei Durchsicht der Kriegsradierungen 1924 wäre man ohnehin auf den Ort Dontrien gestoßen (1. Mappe, Bl. 4). - Diese Ausstellung von 1916 in der Heimat, auf die mich freundlichst O.K. Werckmeister hinwies, wird noch zu thematisieren sein. ${ }^{12}$

Vorerst sollen Selbstzeugnisse von Dix hier wiedergegeben werden, die seine Sicht auf die

nen wie »Finale 17 , Dresden, verwechselte (s. Lehmann 1991, Nr. 41 - die freilich keine Verwundeten darstellt, sondern Auferstehende, siehe unten); vgl. Hans Kinkel, Dix Protokolle der Hölle, Frankfurt/M. 1968, Nr. 40; Dietrich Schubert, Dix, 3. Aufl. 1991, S. 26-29). - Zu Conzelmanns Buch insbesondere die Rezension von Uwe M. Schneede, in: Frankf. Allg. Ztg. vom 26. November 1983.

11 Hans U. Lehmann, OTTO DIX - die Zeichnungen im Dresdner Kupferstichkabinett, Dresden 1991, Nr. 27, die Identifizierung mit Aubérive nicht durch einen Titel von Dix selbst, sondern kombiniert über die Feldpostkarte Gera, Rüdiger Nr. 15, die das granatenzerpflügte Aubérive wiedergibt, ebenso wie das Blatt in Berlin (unsere Abb. 3); eher handelt es sich um Dontrien. - Vergleiche auch die falsche Lesart "Doutrienne« in: Suse Pfäffle (wie Anm. 1), G 1915/12, die die Autorin aber dann noch berichtigte, S. 263. Auch Andreas Strobl in seiner Arbeit über die "Karriere von Dix gibt diese Nr. 243 in der Ausst. 1916 fälschlich als »Vontrieu« an, hat also dabei nur von H. U. Lehmann abgeschrieben: Otto Dix - eine Malerkarriere der 20er Jahre, Diss. München, Berlin 1996, S. 242.

12 Katalog der Galerie Ernst Arnold, Dresden 1916, in der Kunstbibliothek zu Berlin, dank bestem Hinweis und kolle-
Kriegsrealität und seine Gestaltung derselben auf spezifische Weise erhellen.

Ob nun das Dorf Dontrien oder aber Aubérive oder ein anderer Ort - die wochenlangen Granatangriffe und Trommelfeuer zwischen den Fronten der Deutschen und Franzosen an der Linie am Fort de la POMBELLE bei Reims zerstörten die Dörfer jeweils in gleichem Maße. Was entstand, waren Trümmerlandschaften, wie sie Dix später auch in und bei Langemarck/Flandern erlebte (Sommer 1917 und Juli 1918), die er in übernaturalistischer Form zeichnete und die er später in den Radierungen von 1924 in forciert naturalistischer Sicht für die Nachwelt zur Mahnung wiedergab. ${ }^{13}$ Ähnlich dem Blatt in Dresden erscheint das zerschossene Dorf (Dontrien oder Aubérive) in der Zeichnung im Berliner Kupferstichkabinett (Abb. 3).

Der Kommentar von Dix auf seiner Feldpostkarte (FPK) Gera 13/14 vom Februar 1916 kann auch die Blätter in Dresden und Berlin (Abb. 3) erhellen. Dix schrieb:

„In den Trümmern von Aubérive - voll elementarer Wucht sind Granattrichter innerhalb Dörfern. Alles in der Umgebung scheint der Dynamik dieser gewaltigen symmetrischen Trichter zu unterliegen. Es sind die Augenhöhlen der Erde. Was darum herum kreiselt sind irre schmerzlich phantastische Linien. Häuser sind das nicht mehr, niemand glaubt das im Ernst. Es sind Lebewesen von besondrer Art mit eigenen Gesetzen und Lebensbedingungen. Es sind lauter Löcher mit Steinen herum, oder lauter Skelette. Es ist eine eigenartige seltene Schönheit, die da redet .... ${ }^{14}$

Während wir vergleichsweise detailliert unterrichtet sind über die Beteiligung von Dix in den Stellungskämpfen vor Reims/Champagne bis Juli

gialer Hilfe von Otto K. Werckmeister im Herbst 1987; von mir bereits in der 3. Aufl. meiner Dix-Monographie1991, S. 33, publiziert (vergleiche bitte unten Anm. 40).

13 Der Ausdruck übernaturalistisch meint eine Gestaltung des Sichtbaren mittels subjektiver Überformung bzw. Durchdringung. Das sichtbare Objekt oder Sujet wird nicht fotografisch genau (quasi daguerreotypisiert) gegeben, sondern es kommt zur Synthese von Objekt und subjektivem Gestalt-Willen, also von Sehen und Fühlen, von Beobachtung und Erinnerung, gepaart mit Phantasie.

In der Weise hatte Heinrich Heine in "Französische Maler* 1831 geschrieben: "In der Kunst bin ich Supernaturalist«, ein Begriff, den Théophile Gautier 1836, Saint-Beuve 1838 und Ch. Baudelaire 1846 in ihrer Übertragung als "surnaturaliste bzw. "surnaturalisme« übernahmen (vgl. dazu Burkhardt Steinwachs: "Zeitbewegung" als Signatur der Moderne, in: Epochenbewußtsein und Kunsterfahrung, München 1986, S. 259 Anm. 11).

14 Siehe D. Schubert, Dix, 1991, S. 28; O. Conzelmann a.a.O. (wie Anm. 10), S. 127; U. Rüdiger, Grüsse (wie Anm. 4), S. 50 52. - Bei Conzelmann nicht exakt zitiert und derart von $\mathrm{H}$. Lehmann (wie Anm. 11), Dresden 1991, S. 72 abgeschrieben. 
1916, seine folgenden Einsätze in der Sommer- und der Herbstschlacht an der Somme (Juli-August 1916, November-Dezember 1916), ${ }^{15}$ seine Kämpfe in der Sommerschlacht in Flandern (Ende Juli-Mitte Sept. 1917) und über die mehrwöchige Ruhe bei Brügge mit Fahrten ans Meer bei Knocke (vgl. Abb. 1 und die FPK Gera 38 "Dix auf Urlaub«, ein kommentiertes Foto), ${ }^{16}$ ist sein Einsatz an der Ostfront nach der Oktober-Revolution in Rußland zwar gesichert, aber nicht genügend deutlich. Der Militärpaß verzeichnet diesen Einsatz vom 7. Oktober bis 5. Dezember 1917; vom 17.12.1917 bis Anfang Februar 1918 den Waffenstillstand. ${ }^{17}$

Die 1. Maschinengewehr-Kompanie von Dix innerhalb des Inf.Res.Regiments 102 wurde eingesetzt in den Stellungskämpfen zwischen dem NarotschSee und Tweretsch (nördliches Belorußland). Glücklicherweise haben sich unter den Postkarten von Helene Jakob auch solche aus Rußland erhalten, deren Ortsangaben ich bereits 1980 publiziert habe: "Gorodniki« und "Lagoerde - Stützpunkt (Rußland) ${ }^{18}$ hat Dix selbst auf die Rückseiten seiner kleinen Postkarten-Skizzen geschrieben. Die Numerierung 131 und 135, ebenso wie die Daten 18. und 29. November 1917 sind von Helene Jakob, die alle Postkarten numerierte und teils zusätzlich

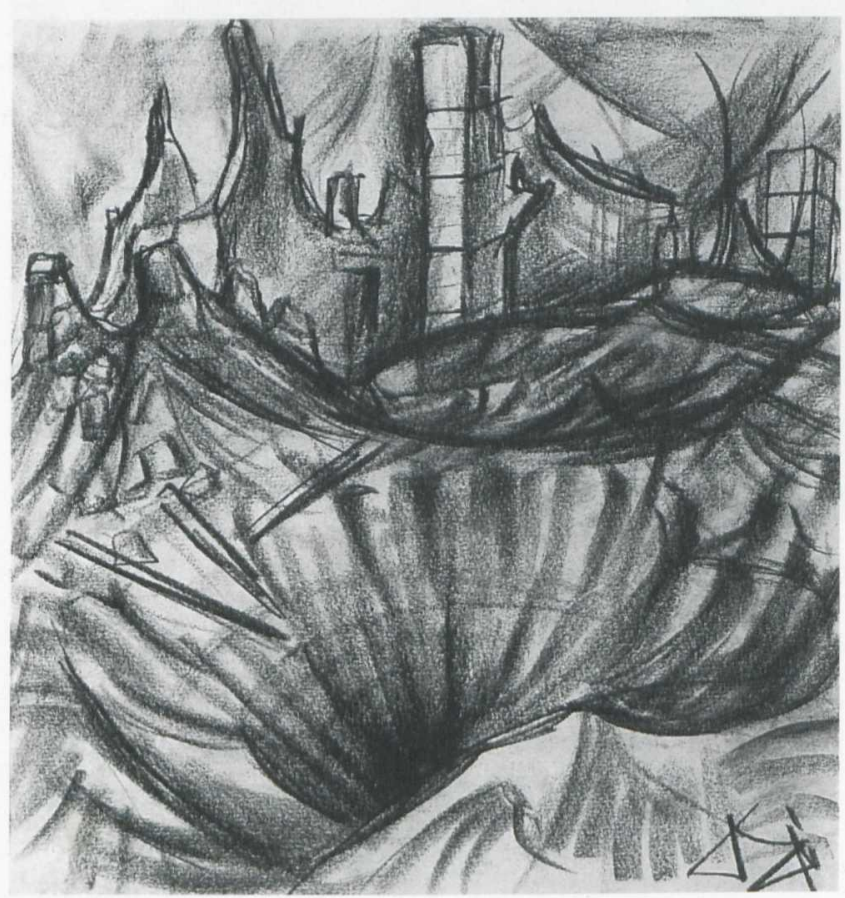

Abb. 3. Otto Dix, Granattrichter, bei Aubérive, Kreide 1916 Staatliche Museen zu Berlin, Kupferstichkabinett

15 Vgl. Militärpaß Nürnberg p. 11; O. Conzelmann (wie Anm. 10) 1983; D. Schubert, Dix und der Krieg, in: Pazifismus zwischen den Weltkriegen, hrsg. von Dietrich Harth/Dietrich Schubert, Heidelberg 1985, S. 185 f. - U. Rüdiger, Grüsse (wie Anm. 4). - D. Schubert, Dix, 1991, S. 23.

16 Vgl. U. Rüdiger, Grüsse (wie Anm. 4), 1991, Nr. 38, S. 100.

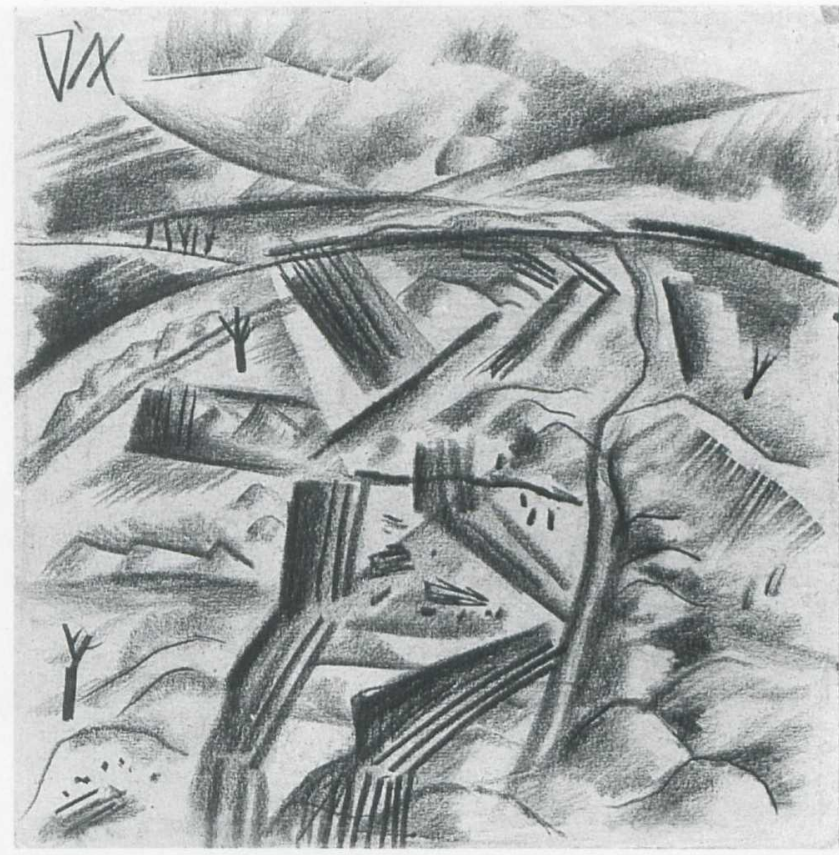

Abb. 4. Otto Dix, Russische Landschaft, Kreide 1917, Galerie der Stadt Stuttgart

datierte (Eingang in Dresden?), hinzugefügt worden. Die futuristische Zeichnung einer ReiterAttacke (Gera 43) wurde von ihr auf den 2.12.1917 datiert. Bereits bald danach schrieb Dix wieder von der flandrischen Westfront (Knocke, Langemarck? FPK Gera 44/45). Dann fehlen bis Mitte April 1918 die Karten an die Dresdner Freundin.

Ob Dix im Winter 1917/18 in Ruhestellung an der Ostfront blieb oder aber bereits im Januar-Februar 1918 wieder in Flandern weilte, wie eine Notiz auf einem Probedruck zu einer Radierung der KRIEG-Mappe 1924. (Blatt 7), "Langemarck Febr. 1918« suggeriert, ist zur Zeit nicht zu klären. Der Militärpaß führt unter Krankheiten und Verwundungen auf: "am 6.2.18 auf Urlaub erkrankt und dem Res.Laz. Gera-Reuß Abt. Schützenplatz überwiesen, am 30.3.18 vom Res.Laz. Gera-Reuß als genesen wieder zur Komp(anie) «. ${ }^{19}$

Ob Dix also im Zeitraum der Waffenruhe vom 6. Dezember 1917 bis zu seiner Erkrankung und Lazarett-Versorgung in Gera ab 6.2.18 in Rußland in Ruhestellung lag oder bereits im Januar 1918 in Flandern weilte, dann bei einem Urlaub in Gera erkrankte, wie der Paß schrieb, kann durch weitere Dokumente z.Zt. nicht beantwortet werden.

Die Geraer Postkarte 44 (Jakob Nr. 144) »Strand bei Knocke am Meer", die die sechste Postkarte

17 Vgl. Militärpaß DIX, Nürnberg GNM p. 16, die gedruckte Übersicht für die Jahre 1917-18.

18 D. Schubert: Otto Dix, Reinbek 1980, S. 22 f., in 3. Aufl. 1991, S. 23.

19 Vgl. Militärpaß Nürnberg, p. 11; ferner U. Rüdiger, Grüsse (wie Anm. 4), S. 23. 


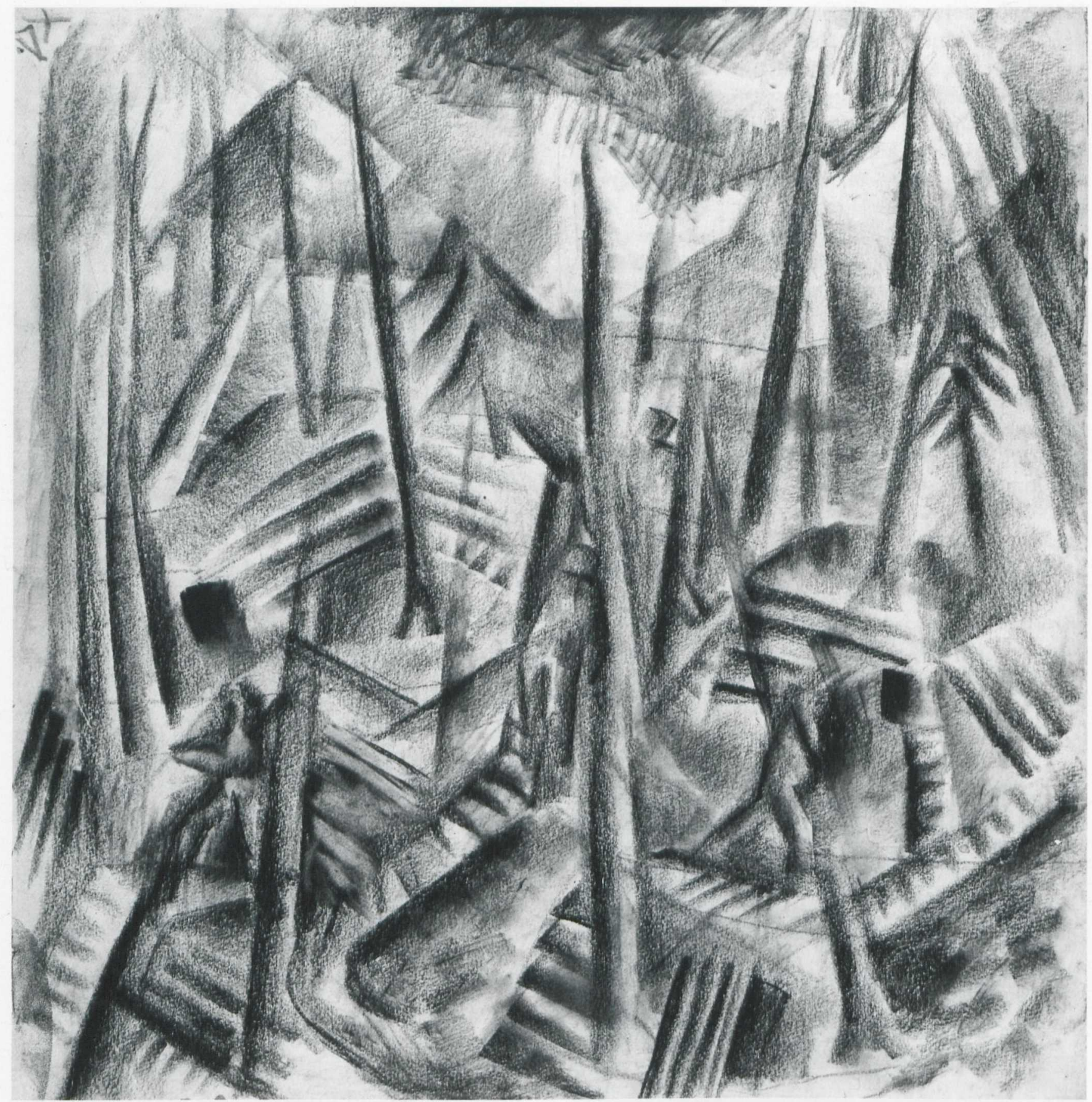

Abb. 5. Otto Dix, Männer im Unterholz (Rußland), Kreide 1917, Privatbesitz

nach den futuristischen Reitern vom 2.12.17 war, trägt leider kein Eingangsdatum der Adressatin. Dafür haben wir andere Kreide- und Kohle-Zeichnungen, die die Monate an der Ostfront beleuchten können: rückseitig beschriftet (wie so oft, aber auch wie so oft, unklar von wem?) ist das Blatt $29 \times 29 \mathrm{~cm}$ der Galerie der Stadt Stuttgart, ${ }^{20}$ "Russische Landschaft« (Abb. 4), d.h. von November-Dezember 1917. Gegenüber den Zeichnungen von 1916 und Anfang 1917 ist in den Rußland-Skizzen die Systematisierung der Zeichnungstechnik fortgeschritten.
Dix arbeitet nicht mehr mit dem Wechsel von Kanten und dynamischen Schwüngen, die die AubériveBlätter und die Somme-Blätter charakterisierten. Deutlicher faßt er nun Teile der Realität in Schraffen zusammen, gliedert die Massen durch das Hell nichtbezeichneter Papierteile. Insbesondere die gewisse Abstraktion, weniger Details zu zeichnen, weniger genau die Komplexheit der Realität zu erfassen und dafür das bewußt stehengelassene Hell des Grundes zu aktivieren, charakterisiert den Stil von Dix seit Sommer 1917 (Badende Kompanie bei

20 Bestandskatalog Galerie der Stadt Stuttgart, bearb. von Brigitte Reinhardt, Stuttgart 1989, Nr. 74. 
Knocke, Abb. 1) und besonders dann im Herbst 1917 in den Rußland-Blättern. Zu großer Meisterschaft steigert Dix diesen Zeichenstil, indem er stärker die kubistisch-futuristischen Facetten von Bewegtheit berücksichtigt und in der Abfolge von Parallel-Schraffen die Simultaneität der Bewegungen von Menschen und Natur bzw. Mensch und Ereignissen anschaulich wirksam macht, z.B. in der Zeichnung, die Conzelmann als »Drei Männer im Gehölz» führte. ${ }^{21}$

Das charakteristische Format (beinahe quadratisch, $40 \times 38,5 \mathrm{~cm}$, Privatsammlung, Abb. 5) gibt vom erhöhten Standpunkt den Blick in ein Unterholz bzw. ein Waldlager wieder; die zahlreichen Holzstege werden von Dix gleichsam wie Fächer gezeichnet und zwischen die. Baumstämme in Vorder-, Mittel- und Hintergrund eingebaut. Es bewegen sich vier Männer im Wald, die lediglich schemenhaft abstrahiert erkennbar sind. Im Falle dieser Kriegszeichnung wird die nähere Bestimmung des Blattes ermöglicht durch die kleine Postkarte 42 in Gera, die die Komposition jedoch steiler baut, die aufgefächerten Motive zusammenschiebt, nur drei Männer einfügt und im Zentrum die Tanne deutlicher und dunkler akzentuiert (Abb. 6). Mit der Betitelung dieser Postkarte »Im Stützpunkt Lagoerde (Rußland) « und dem Jakobs-Datum 29.11.1917 ist die große Zeichnung hinlänglich eingeordnet.

Künstlerisch signifikant wird die Dixsche Verarbeitung von Erlebnissen des Krieges, Kampfszenen, Naturzerstörung und von Motiven der Menschen in Natur oder in Trümmern immer dann, wenn wir verschiedene Versionen einer Gestalt-Idee besitzen. Dies ist der Fall bei dem Blatt in Dresden und in Privatbesitz aus dem Herbst 1917, das umseitig mit »Finale« bezeichnet ist, das nicht Verwundete am Abend (so Conzelmann und Lehmann) zeigt, sondern Auferstehende, die ihre Köpfe und Arme zu einer übergroß erscheinenden Sonne recken. Das Dresdner Blatt wurde von Conzelmann verwechselt mit der 2. Version, und er unterstellte mir eine „Umfrisierung « von Bildtiteln bei Dix. ${ }^{22}$

Auch für das vehemente Blatt "Volltreffer" in Stuttgart, das in kubistischem Formenwirbel den Tod eines Mannes durch eine Granate zeigt, tauchte jüngst eine 2 . Version von $35 \times 41 \mathrm{~cm}$ auf (Abb. 7 ), ${ }^{23}$ die die kubistisch verfremdete Signatur rechts unten zusammen mit dem Datum »17 1 gibt.

21 Otto Conzelmann, Der andere Dix, 1983, Abb. 187.

22 O. Conzelmann, 1983, S. 12 und Abb. 119 die 2. Version in Privatbesitz, die Conzelmann mit dem Dresdner Blatt verwechselte! - Vgl. dazu meine Besprechung des Buches in: Kritische Berichte, 12. jg., 1984, Heft 1, S. 84-94, Abb. S. 86; Hans U. Lehmann (wie Anm. 11), 1991, S. 82.

23 Das Blatt „Volltreffer I« in Stuttgart, Gal. d. Stadt (Bestandskatalog 1989, Nr. 69), dazu die 2. Version Kreide $35 \times 4.1 \mathrm{~cm}$,

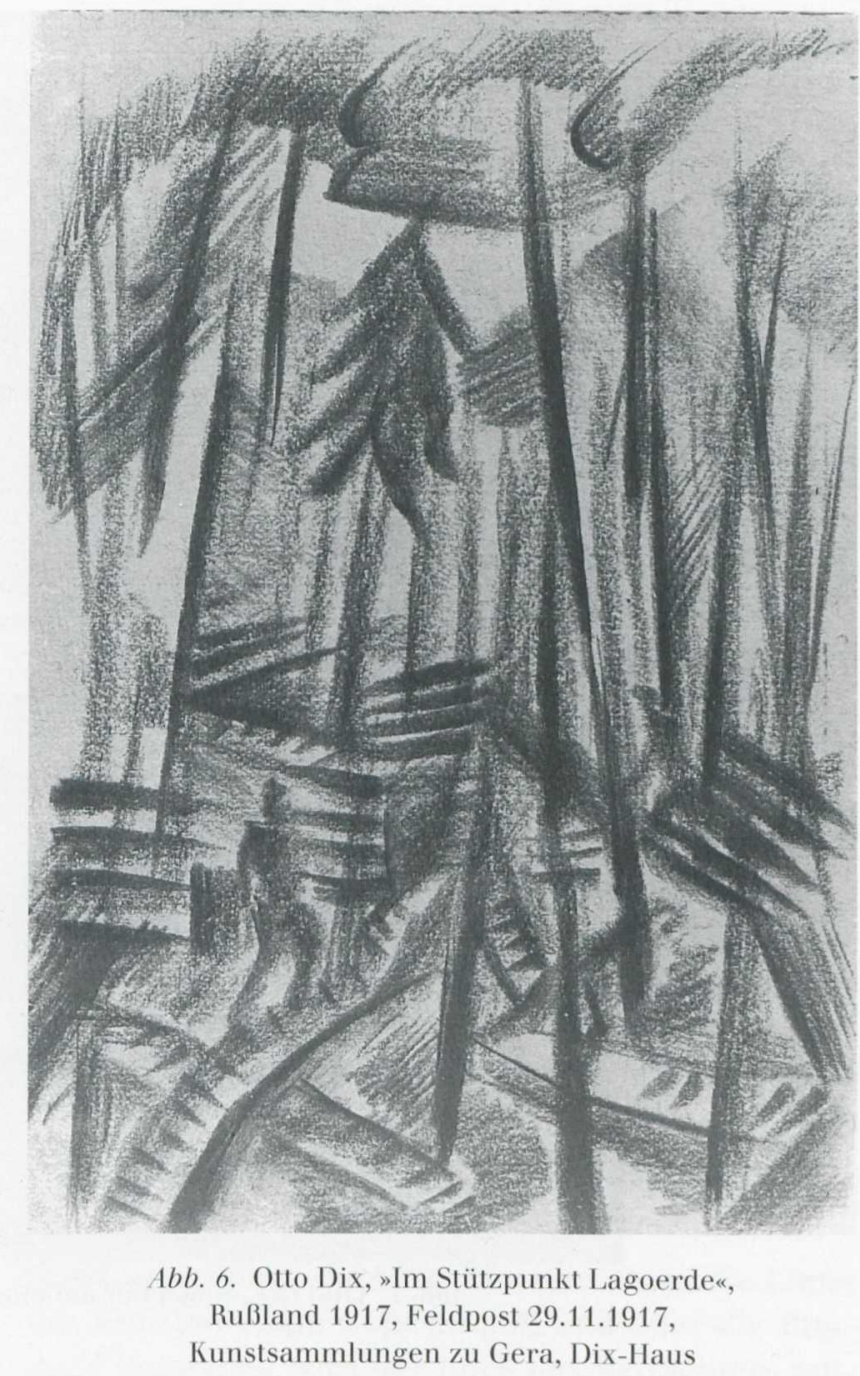

Es fällt auf, daß Dix auch in anderen Fällen für bestimmte Stoffe verschiedene Varianten oder Versionen zeichnete: bei den badenden Soldaten am Meer die Feldpostkarte von August 1917 (Gera 39), die große bildmäßige Zeichnung »Knocke 17 «, und er führte eine Gouache mit wenigen Farben auf hellocker Malgrund aus (Galerie Valentien, Stuttgart, 1990/91). Zu Recht hat U. Rüdiger 1991 zum Verhältnis von großer Zeichnung und Postkarte geschrieben: »Hier wird deutlich, daß die auf Postkartenformat reduzierten Kohlekompositionen hin und wieder zeichnerische Eleganz, expressive Kraft und suggestive Dichte einbüßen, eben das, was die überragende Qualität der großen Kreide- und Kohleblätter aus dem Ersten Weltkrieg ausmacht. ${ }^{24}$

datiert (vgl. Katalog der Berliner Auktion Villa Grisebach, 21, Berlin, November 1991, Nr. 46). - Ich beleuchte dieses Phänomen der doppelten Zeichnungen auch in meinem Beitrag: Otto Dix zeichnet im 1. Weltkrieg, in: KULTUR und KRIEG, hrsg. von Wolfgang J. Mommsen, München 1995, S. 179-193.

24. U. Rüdiger, Grüsse (wie Anm. 4), S. 18. - Dies., in: Kat. Dix (wie Anm. 2), Stuttgart-Berlin 1991, S. 56. 


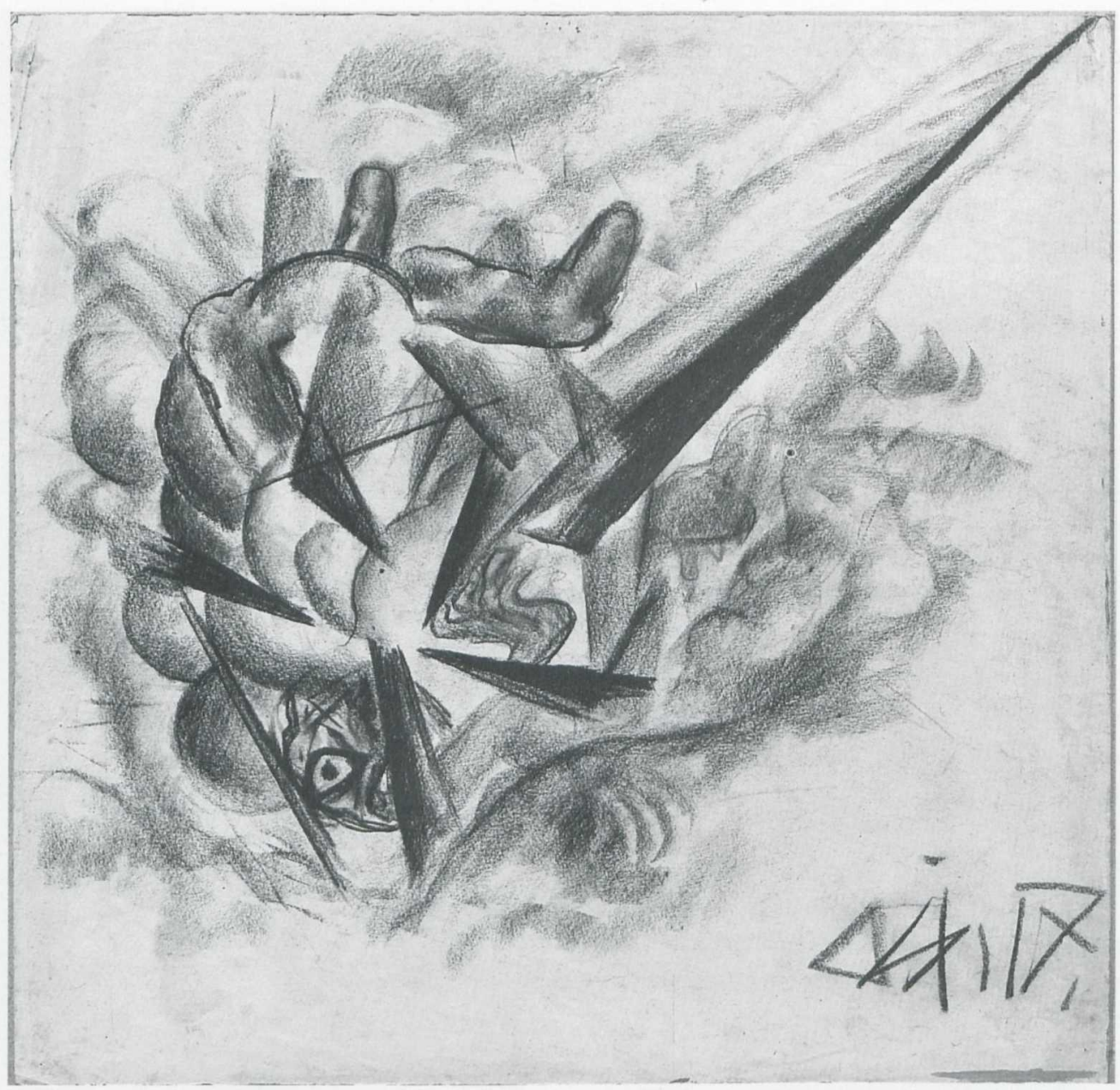

Abb. 7. Otto Dix, Volltreffer auf einen Soldaten, Kreide 1917, Privatbesitz

Doch scheint mir trotz dieser Beobachtung und dem möglichen Qualitätsunterschied zwischen Postkarte und großer Zeichnung erst in der wechselseitigen Behandlung aller Versionen und Varianten sowohl die enormen Willenskräfte des Künstlers und Soldaten Dix im Kriege anschaulich als auch das dialektische Vorgehen als Kondition des Arbeitsprozesses, d.h. letztlich die Aneignung aller Seiten des Kriegserlebnisses erhellt. Niemand sonst hat innerhalb und während des Krieges derart gearbeitet wie Dix. Und er hat erstaunlicherweise eben mit viel Glück, wie der Halsschuß vom 8.8.1918 bei Lestrem/b. Lille zeigt - zugleich überlebt. Denn schließlich waren die Leutnants als die Kompanieführer und die Unteroffiziere und Feldwebel als MG-Truppführer wie Dix diejenigen, die bei den Angriffen am schnellsten im Gegenfeuer fielen.

25 Otto Dix hier zitiert nach Diether Schmidt, Dix im Selbstbildnis, Berlin 1978, S. 237 und 239. Es handelt sich um die Dix-Gespräche auf der Schallplatte des Erker-Verlages in St. Gallen 1963. - Zu Dix und Friedrich Nietzsche vgl. D. Schubert, Dix, 1. Aufl. 1980, S. 56 f.; Otto Conzelmann a.a.O.
Dix muß quasi besessen gewesen sein von dem gleichsam sdionysischen, Willen, den ihm Nietzsches Lektüre introjiziert hatte, durch diesen Krieg wie durch ein »Feuer« durchzumüssen, zu kämpfen, nicht um zu töten (morden), sondern um zu überleben, Handgranatenwerfen zu üben, um zu überleben (wie Otto Griebel berichtete) und eben auch zu zeichnen, um die Sache zu bezeugen, also künstlerisch gestalten, um zu demonstrieren: „So ist das gewesen und nicht anders. ${ }^{25}$

Dix hätte mit Max Beckmann - der dies im Kriege von der Ostfront 1914 an seine Frau Minna Tube schrieb - beteuern können: "Ich habe gezeichnet, das sichert einen gegen Tod und Gefahr. ${ }^{26}$ Eine gewisse vitale Faszination an den Erlebnissen des Krieges mag Beckmann und Dix gemeinsam sein, freilich immer in psychischer Ambivalenz teils Grauen, teils Faszination und Friedenswunsch.

1983 (wie Anm. 10); Schubert, Dix, 3. Aufl. 1991, S. 54-58; Sarah O'Brien-Twohig, Dix and Nietzsche, in: Catalogue Exposition OTTO DIX, The Tate Gallery London 1992, pp. $40 \mathrm{f}$.

26 Max Beckmann, Briefe im Kriege (1916), Neuausgabe München 1984, S. 13 (3. Oktober 1914); vgl. oben Anm. 5. 
Beckmann pries das »wilde Leben, das da entfesselt wurde" (10. März 1915) und schrieb - trotz des Erlebnisses eines bedrohlichen Granatangriffs der Engländer Anfang Mai 1915 bei Ypern - wieder: »Eine wilde Welt. Wie fern ist der Friede. ${ }^{27}$

Dagegen notierte Dix trotz seines extraordinären Überlebenswillens und seines außergewöhnlichen Arbeitswillens als Maler auf Feldpost am 6. Juni 1916 am Fort de la Pombelle (Reims) auf einer Postkarte, die den MG-Stand wiedergab: „Das ist unser betonierter MG-Stand, durch die Schlitze wird geschossen. Hoffen wir, daß bald Friede wird! Viele herzliche Grüße Via samideano (Ihr Gleichgesinnter) DIX." 28

Der angeblich vom Krieg begeisterte Dix (so Conzelmann) wünschte sich also bereits nach eineinhalb Jahren Frieden, mußte diesen Krieg aber noch bis November 1918, also eine lange Zeit, durchstehen. Sorgen und melancholischen Friedenswillen reflektiert m.E. das kleine, dicht gezeichnete Selbstbildnis auf einer Postkarte an die Jakob (Kat. Gera Nr. 23), das ohne Zweifel an das berühmte DÜRER-Selbstporträt in Erlangen von um 1492 anschließt. $^{29}$

Helene Jakob hatte Dix im Januar 1916 DürerDrucke geschickt, "möglicherweise war eben jene Dürer-Zeichnung dabei« (U. Rüdiger). Dix sitzt im düsteren Unterstand am Tisch schreibend und zeichnend; der Rechtshänder beobachtet sich in einem Spiegel und gibt sich direkt - ohne Deformationen aus Phantasie ${ }^{30}$ - wieder (Abb. 8).

Der Zeichenmodus ist nicht kubistisch oder futuristisch, sondern entspricht formästhetisch und gehaltsästhetisch den Kriterien des REALISMUS, d.h. die plastischen Massen des Wirklichen werden im Wesentlichen des Hell-Dunkel gegeben. Das Charakteristische des sichtbar Wirklichen wird mit vereinfachenden Mitteln dargestellt, leicht abstrahiert, kein Trompe-l'œil, keine fotografische Genauigkeit, sondern Verzicht auf das Unwesentliche, also Konzentration auf das Wesentliche in einer dichten Komposition, die natürlich mit der traditionellen Bildwirksamkeit rechnet. Die Gegenstände im Vordergrund, das Licht auf dem Papier, auf dem die

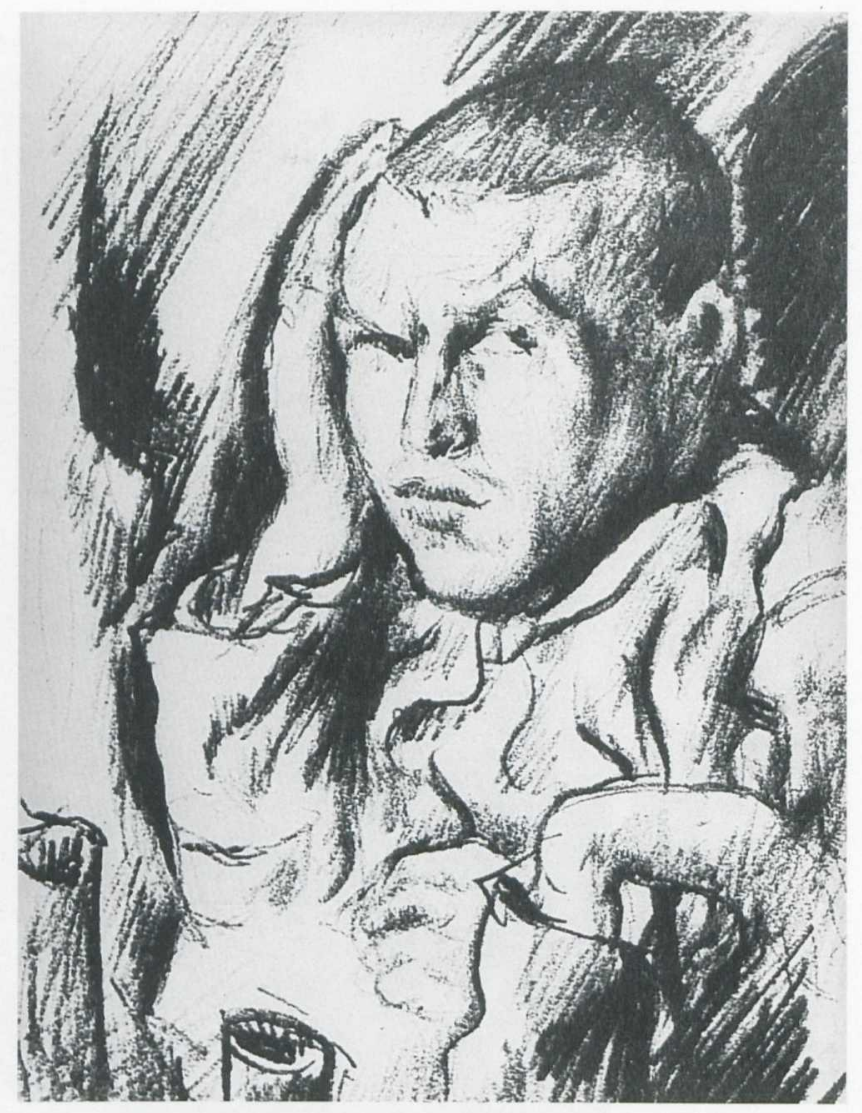

Abb. 8. Otto Dix, Selbstbildnis im Unterstand, Feldpostkarte 20.3.1916, Kunstsammlungen zu Gera, Dix-Haus

Hand ruht, leiten zur Mitte hin; dort steigt die Linke des Künstlers zum Kopf hinauf, und über die Rundung desselben wird der Blick des Betrachters mittels der Augenrichtung ins Zentrum von Form und Gehalt geführt. Von Dürers Selbstbildnis weicht Dix darin ab, daß er die Seiten, d.h. die Blickwinkel verändert. Auch sucht er nicht die Übergenauigkeit Dürers. Dix begnügt sich mit der Verdichtung aufs Wesentliche in der Modellierung des Kopfes mit weichem Blei im Dunkel und im Hellen.

Der Zeichenduktus dieses kleinen Selbstporträts - und das stützt visuell die Datierung in März 1916, nicht 1917 - arbeitet noch mit den Rundungen und Kurvungen der Striche, mit gekrümmten Schraffen und Verdoppelung der ondulierenden Konturen, - was den Zeichnungen von um 1915/16
27 Max Beckmann, Briefe im Kriege (1916), 1984, S. 37. Zum Kontext der Künstler im Krieg vgl. Christian Lenz und Dietrich Schubert in: KULTUR und KRIEG, hrsg. von W.J. Mommsen (wie Anm. 23)

28 Feldpostkarte Gera Nr. 31, vgl. D. Schubert, DIX (1991), S. 28. - U. Rüdiger, Grüsse (wie Anm. 4), S. 86.

$29 \mathrm{Vgl}$. D. Schubert, Rezeptions- und Stilpluralismus in den frühen Selbstbildnissen von Otto Dix, in: Beiträge zum Problem des Stilpluralismus, hrsg. von Werner Hager, München 1977, S. 218 Abb. 24-25; U. Rüdiger (wie Anm. 4), S. 12 und S. 70, Nr. 23. - Die Angabe 1917 in meiner Dix-Monographie 1991, S. 23, muß korrigiert werden; bei Otto Conzelmann, DIX-Handzeichnungen, 1968, 46 und: op.cit. 1983 (Anm. 10),
S. 78 und Abb. 96 auf 1917 datiert. - Unter dem Titel »DIXNeuland " hat Rainer Beck 1992 Ergebnisse meiner älteren Beiträge ausgeschlachtet, auch den Dix-Text von 1947 für eine Ausstellung in Gera hatte ich bereits 1980 in der 1. Aufl. meines Büchleins S. 122 publiziert (R. Beck, in: Katalog DIX die Friedrichshafener Sammlung, hrsg. von Lutz Tittel, Friedrichshafen 1992, S. 15f.).

30 Jurij Lotman unterschied ,Déformation a aus Bedingtheit und solche aus Phantasie (Aufsätze zur Theorie und Methodologie, hrsg. von K. Eimermacher, Kronberg 1974, S. 15-20). - Auch in Emile Zolas Kunsttheorie spielte der Begriff der déformation bereits um 1864/66 eine zentrale Rolle. 


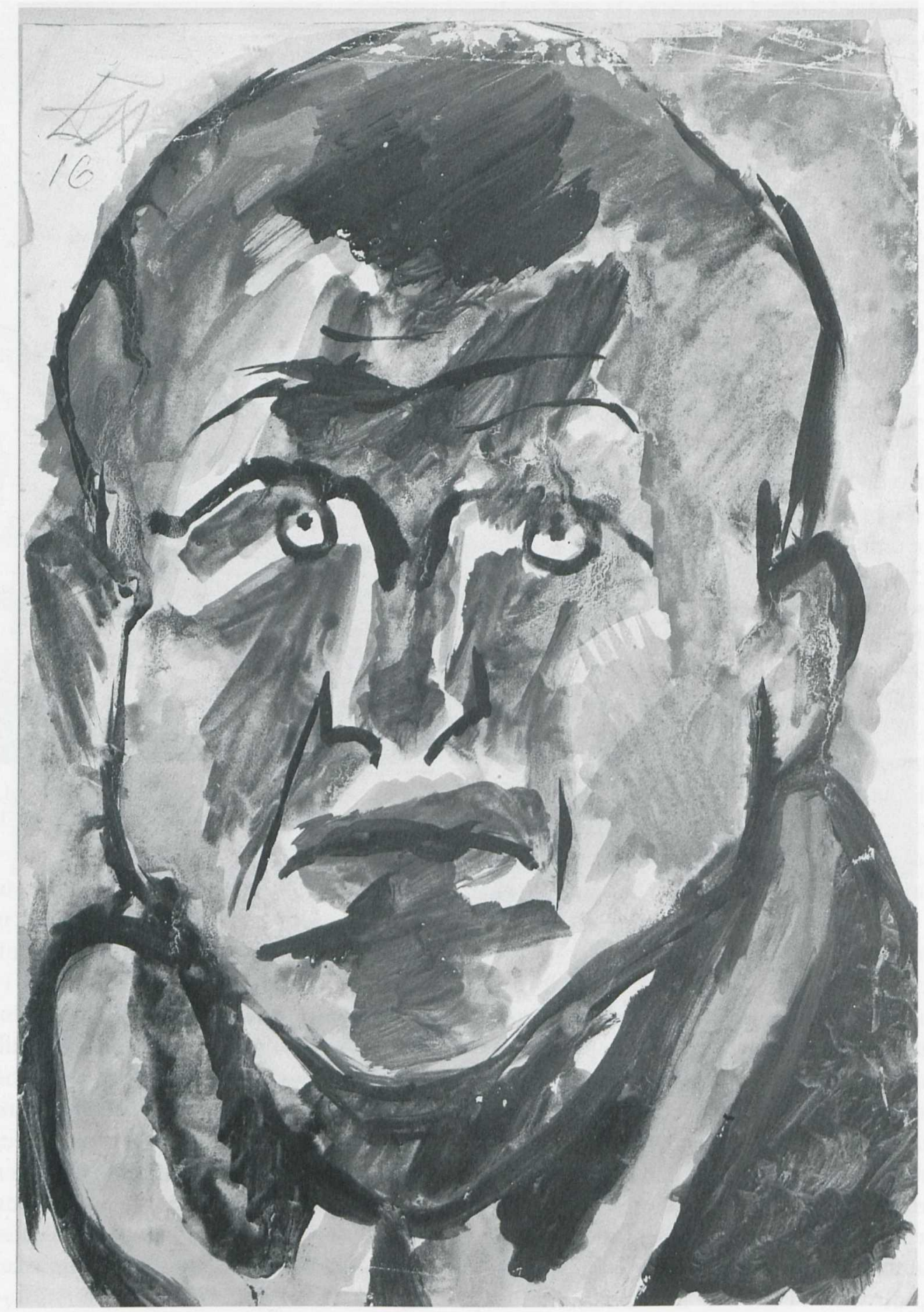

Abb. 9. Otto Dix, Selbstbildnis, Gouache/Aquarell 1916, Privatbesitz

entspricht. Der stärker formsystematisierende Zeichenstil des Jahres 1917 - wie wir sahen - wird die Dinge mehr in prismatische Formsukzessionen zwingen und die Kubenbildung präferieren. Im Jahr 1916 geht Dix noch stärker vom Wesentlichen der beobachteten Dinge aus; später denkt er in quasi ,futuristischen`Gestaltungselementen.

31 Die Gouache dieses Selbstporträts von 1916 war die Nr. 377 in der Auktion von Hauswedell + Nolte, Hamburg 1974; nicht bei Diether Schmidt a.a.O. (wie Anm. 25) 1978; - bei Su-
Zieht man andere Selbstbildnisse heran, so wird dies deutlich: Die Gouache des frontalen Selbstporträts (Abb. 9), die Dix 1916 datierte, aber erst ca. 1960/62 signierte, ${ }^{31}$ arbeitet ebenso wie die Kriegszeichnungen von 1916 mit kurvenden Formen und Strichpartien. Das Mittel des Stehenlassens des Malgrunds für die hellen Formteile wird

se Pfäffle, Werkverzeichnis (wie Anm. 1) 1991, A 1916/4 als Aquarell (?). 
aber bereits geübt. Wie die Entwicklung der Formensprache insgesamt verlaufen sollte, wird anschaulich beim Blick auf die meisterhafte Kreidezeichnung des Selbstporträts im Halbprofil nach rechts, mit geschlossenen Augen unter dem Schirm der Uniformmütze. Dix stellt sich in einem merkwürdigen Habitus der Verschlossenheit nach Außen dar, d.h. im Ausdruck innerer Konzentration. Die Kleidung ist nicht die im Kampf, sondern eher die Ausgangsuniform, wie auch auf dem erwähnten Foto »Dix im Urlaub« von August 1917 in Brügge (FPK Gera Nr. 38).

Im Gegensatz zur Gouache werden alle Formen in ein System von Kuben überführt, das zwar die Radikalität der Zeichnung "Badende Soldaten« bei Knocke (Abb. 1) oder des Stuttgarter Blattes "Zwei Schützen« von 1917 noch nicht erreicht hat, aber das Ziel des Kunstwollens ist sichtbar. Augen, Nase, Mund, Kinn, Ohren und Schirm werden zusammen mit den Schatten an Hals und Wangen in kantige Formen überführt, die an die Sichtweise eines expressionistischen Bildhauers gemahnt. ${ }^{32}$ Auch die Signatur rechts oben reflektiert diesen Formwillen: das D wird zu einem Dreieck, I und X werden kraftvoll betont (Abb. 10).

Das andere Selbstbildnis mit Uniformmütze als Unteroffizier (Beförderung bereits am 1.11.1915) im Kupferstichkabinett Berlin, das den MelancholieHabitus des aufgestützten Kopfes mit einem extrem sorgenvollen Blick und einem brutal willenmäßigen Mundausdruck - als Raucher - kombinierte, ist links oben 1917 datiert. Da es noch stärker mit runden, konvexen Formen arbeitet, dürfte es in den Anfang des Jahres gerückt werden müssen, vor das Selbstporträt mit geschlossenen Augen (Abb. 11).

Die Unentschiedenheit des Übergangs von 1916 auf 1917, die Entwicklung zu einem stärker formsystematisierenden Zeichenstil im Laufe 1917 bis zur Rußland-Zeit offenbart auch eine noch nicht publizierte Kreidezeichnung (Privatbesitz), die von erhöhtem Standort den Blick in eine Trümmerlandschaft nach den Zerstörungen monatelanger Granatenkämpfe wiedergibt. Im Hintergrund erkennen wir die Reste einer Ortschaft; in Mittel- und Vordergrund erstreckt sich das umgeackerte Land, das durch einen dunkel gezeichneten Graben in halb runden, halb kubischen Formen unterbrochen ist.

32 Das Blatt "Selbstporträt mit Uniformmütze« (Kreide $43 \times$ $34 \mathrm{~cm}$, rechts oben signiert DIX) bei Hans Kinkel, Protokolle der Hölle, 1968, Nr. 26. - D. Schubert, Dix und der Krieg, in: Pazifismus (wie Anm. 15), 1985, Abb. 5, S. $185 \mathrm{ff}$. - Diese Zeichnung war ehem. im Besitz der Galerie Klihm, München, jetzt S. Sabarsky, siehe Katalog OTTO DIX, Kunsthalle Berlin 1987, Nr. 70 und Katalog Dix - die frühen Jahre, hrsg. von Serge Sabarsky, Wanderausst. 1992-93, Nr. 12 - aber falsch datiert auf 1916. Alle Stilmerkmale sprechen für 1917, so wie "Fruchtschale« Nr. 26 in diesem Katalog sicher zum gleichna-

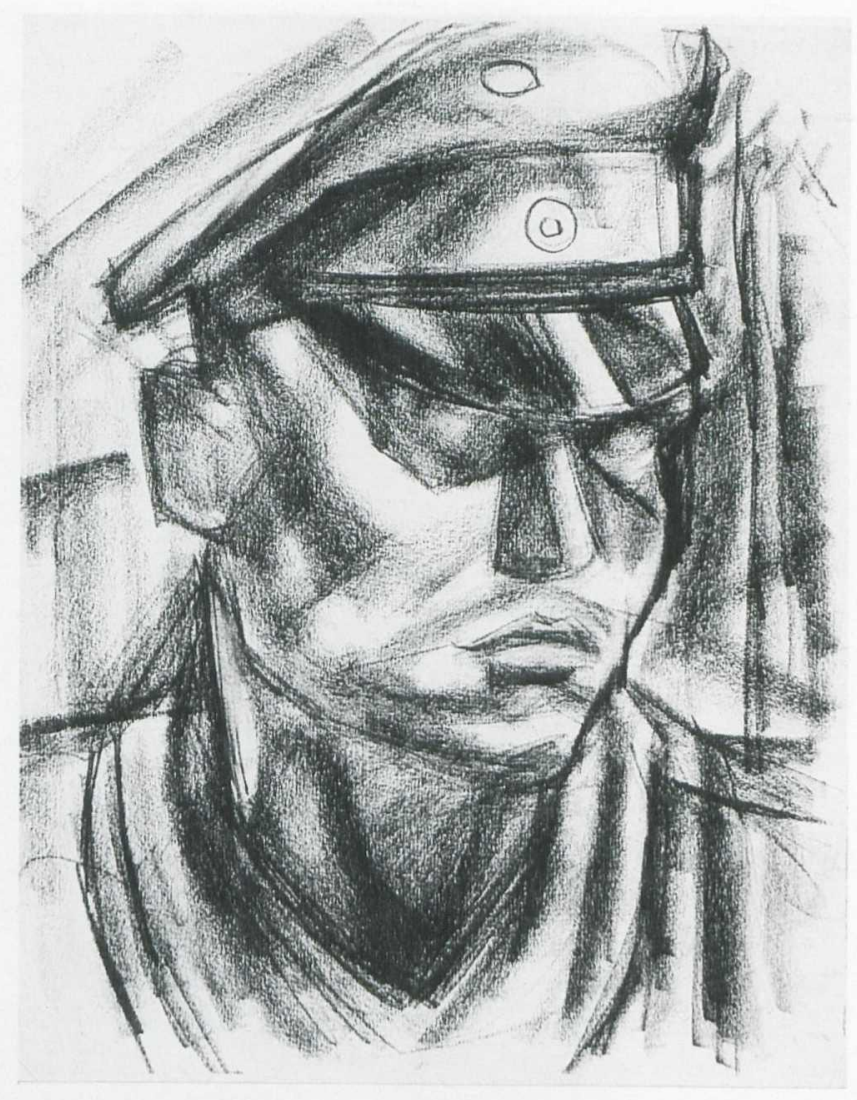

Abb. 10. Otto Dix, Selbstporträt mit geschlossenen Augen, Kreide 1917, Privatbesitz USA

Der sich in die Tiefe zu verjüngende Graben wirkt wie eine brutale Wunde, die spitz zuläuft. Im Hintergrund mündet der Graben in ein zerstörtes Gebäude, das wie ein dämonisches Antlitz einer Riesenplastik den Horizont verstellt. Der Graben scheint dort in das Maul dieses amorphen Kopfes zu münden. Die Signatur des Blattes ist bereits kubistisch verkürzt bzw. forciert zu spitzen DX, d.h. der Künstler zieht das I seines Namens ins X hinein. Das zweite kleinere x könnte als 17 gedeutet werden, - eine Datierung, die sich ohne Zweifel von den Formen her auch ergibt.

Rückseitig ist das Blatt (Abb. 12) - von anderer Hand? - beschriftet "Graben zwischen Trümmern, bei Amiens/Somme «. ${ }^{35}$ Amiens, durch das die Somme verläuft, liegt südlich von Arras, und Dix war nach den Eintragungen im Militärpaß im März 1917 im Artois, genauer gesagt südlich von Arras eingesetzt worden. ${ }^{34}$

migen Blatt mit Datum 191'5 gehört. - Dix wird seit Jahren radikal vermarktet, aber man bemüht sich weniger um eine feinere Unterscheidung der künstlerischen Mittel.

33 Die Amiens-Zeichnung Kreide auf ockerfarbenem Papier $29 \times 28,5 \mathrm{~cm}$ bislang nicht publiziert (Privatbesitz).

34 Vgl. Militärpaß, Nürnberg GNM, p. 16; U. Rüdiger, Grüsse (wie Anm. 4), S. 22; Schubert (wie Anm. 2) 1991, S. 23. - Andrea Hollmann/Ralph Keuning, Biographie DIX, in: Katalog OTTO DIX zum 100. (wie Anm. 2) 1991, S. 15, genauer die Angaben zu den Kriegsstationen bei U. Rüdiger. 


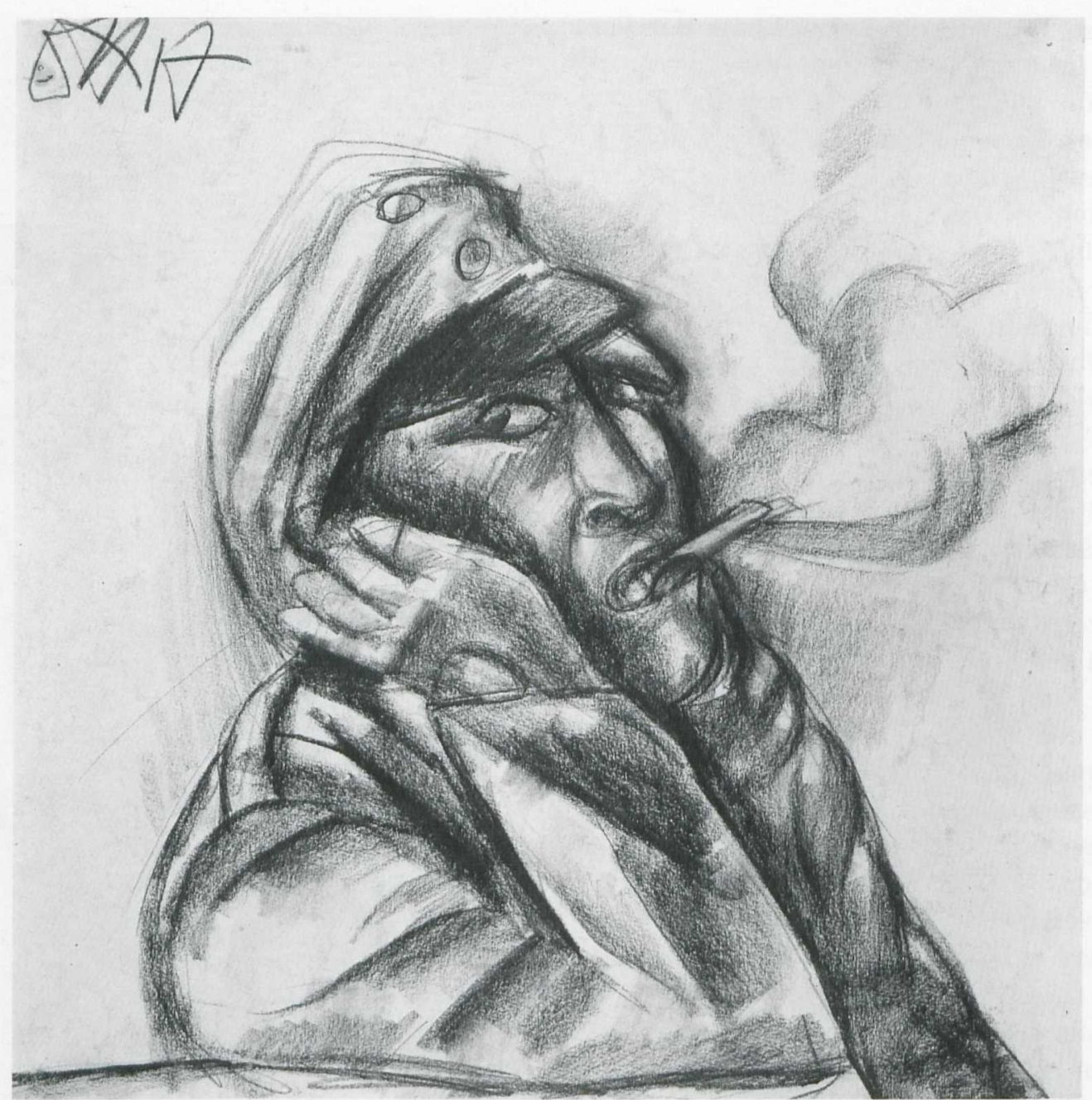

Abb. 11. Otto Dix, Selbstbildnis rauchend, Kreide 1917, Staatliche Museen zu Berlin, Kupferstichkabinett

II.

Mit dieser Kreidezeichnung sei übergeleitet zu der noch unbekannten Gouache, die in leuchtenden Farben eine schwer zu deutende Landschaft wiedergibt (Abb. 13). Das Gemälde von $40 \times 42 \mathrm{~cm}$ befindet sich in Darmstadt (Privatbesitz) und war im Jahre 1961 bei Lempertz in Köln versteigert worden. ${ }^{35}$ Auf dem beinahe quadratischen Format der ockerfarbenen Pappe ist mit sparsamen Mitteln eine riesige helle Säule in einer Landschaft aus Gräben, Mauern, dichten Baumstämmen und Gebäuden im Hintergrund ins Zentrum gesetzt. Dix läßt das Ocker des Malgrundes auffallend stark stehen, um es für die Bildwirkung zu aktivieren. Das Spätherbstliche der Natur wird somit suggeriert. Die Baumstämme sind kahl, wie schwarze Krallen heben sie sich in ihrer Gestik vom hellen Himmel ab, der mit einigen Blaufeldern markiert ist. Die Nei-

35 Auktion 465 bei Lempertz, Köln 1961, lot 109 „Säule in Landschaft« (mit Dank für freundliche Hilfe an H. Hanstein, gung der Stämme ergibt die Zentrierung der Komposition auf das Hauptmotiv der großen Säule hin. Sie vereint in sich alle diejenigen Buntfarben, die Dix auch in der Landschaft anwendet: Weiß, Karminrot, Grün, Königsblau, Schwarz. Die Bildgestalt der Baumstämme erinnert auf den ersten Blick an die in Rußland entstandene Zeichnung der »Männer im Unterholz« (Abb. 5), auch die fächerhaften Formen in der Himmelszone. Doch steht die Darstellung der Gebäude und die Umbildung des Vordergrundes in eckig geknickte Formen mit Binnenschraffuren deutlich der $1917 \mathrm{zu}$ datierenden Kreidezeichnung in Privatbesitz (Abb. 12) nahe. Man erkennt bei genauem Sehen eine Überführung des Zeichenstils der Kreide quasi in die Mittel der Gouache. Auch eine leichte Physiognomisierung der Gebäude ist ähnlich. Die Dämonisierung der Trümmerlandschaft ist aber nicht derart deutlich. Dafür ist mit der riesigen Säule ein Element ge-

Köln, und den Besitzer der Gouache); wie andere Gouachen nicht im Werkverzeichnis von S. Pfäffle 1991 (Anm. 1). 


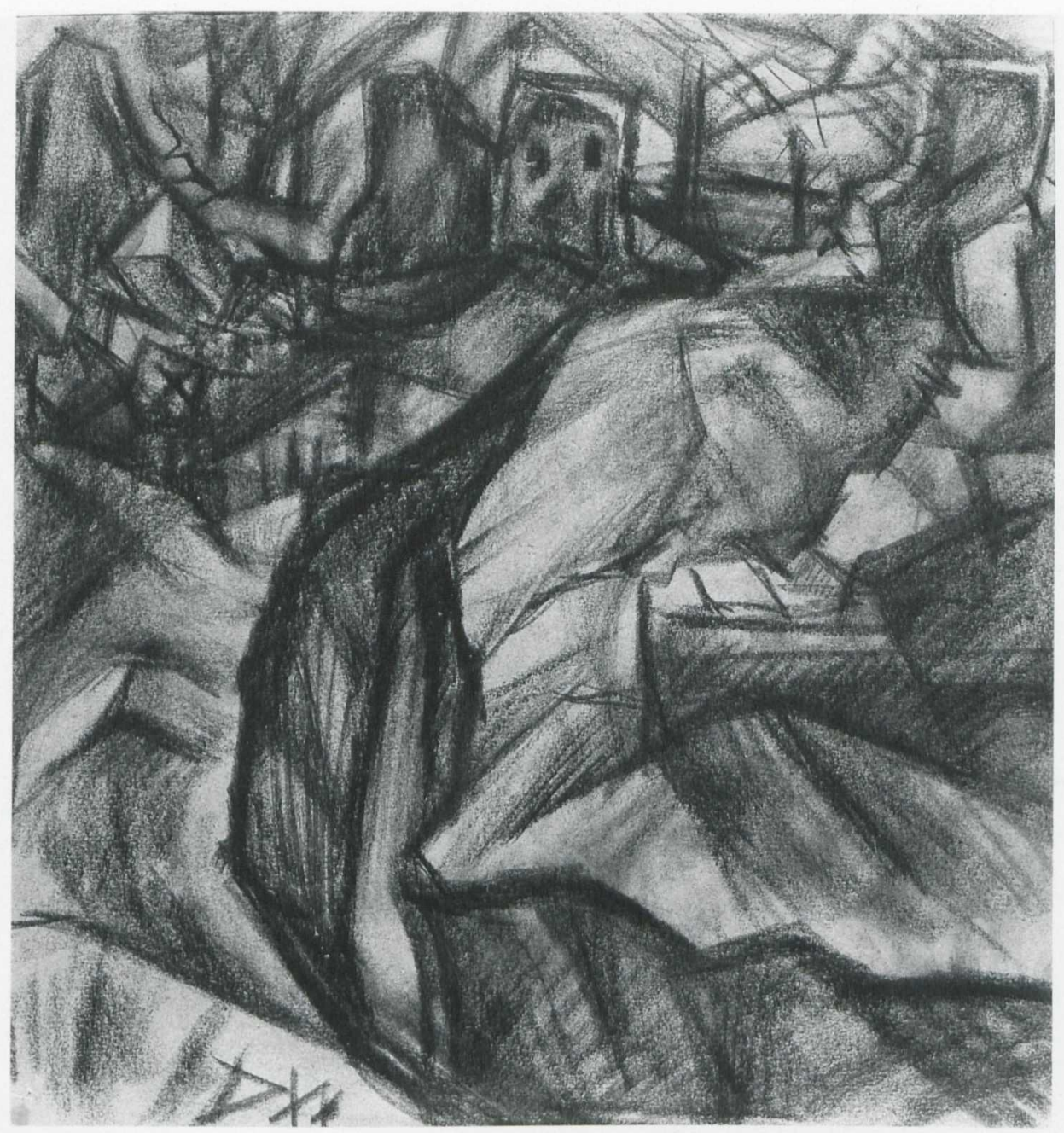

Abb. 12. Otto Dix, Gräben bei Amiens/Somme, Kreide auf braunem Papier, 1917, Privatbesitz

zeigt, das für die Deutung einen enigmatischen Rest schafft, denn die Säule changiert in der Optik von Dix zwischen überirdischem Relikt, Bauwerk, Waffenstück und anthropomorphem Objekt. Dix muß selbst primär überrascht gewesen sein, eine solch blendend weiße Säule großen Formats in einem Schloßgarten erblickt zu haben, so daß er sie im Malprozeß 3 als fremd und rätselhaft, mit dem Kopfteil im Himmel sie beinahe mystifizierend, festhielt.

Hier wird die Arbeitsweise von Dix in eine gleichsam futuristische Dimension gelenkt. Hatte Dix in vielen Zeichnungen 1917, besonders im letzten Jahresteil, die futuristische Formensprache der Dichte der Bewegungsphasen für die Wiedergabe von Simultaneität genutzt, so stoßen in manchen Blättern und Gouachen diese Bildmittel der italienischen Futuristen in eine Dimension des Inhalts bzw. des Gehaltes durch. Die hohe Meisterschaft und die Suggestivität der Dixschen Zeichenweise mit Hilfe futuristischer Formen führt zu zwingenden Bildgestaltungen, die über die beobachteten Bewegungen und Dynamiken in der Natur und im Kampfgeschehen zu neuen Bild-Gestalten führen.
Anders gesagt: die gefundene Bild-Gestalt ist das Ziel des Willens, mit den künstlerischen Kräften die psychischen Emotionen, Hilflosigkeiten, Ängste und Erlebnisse des totalen Eingespanntseins in eine zerstörerische Kampfmaschinerie zu relativieren. Dix als besessen arbeitender Künstler, der in den Kampfpausen nicht nur säuft, sondern auch malt, sucht sich in diesem Gestalt-Willen existentiell als aktives Wesen zu behaupten. Bleiben die anderen Soldaten kleine Räder innerhalb des Kampf- und Tötungsgeschehens, in der Dynamik des Kreislaufs von Töten und Getötetwerden, dem Dix eigentlich ebenso unterliegt, so erhebt er sich dennoch als schaffender Beobachter und Maler-Zeichner auf den Stand eines reflektierenden Subjekts, das sowohl sich selbst bewahrt, ja sichert, als auch die wahre Reportage dieses Krieges liefern wird, d.h. das, was dieser Krieg war: das ununterbrochene Töten für soziale Abstraktionen (wie "Vaterland»), die Zerstörung der Natur als Grundlage des Menschen, die Dämonisierung der Welt durch diesen technisch forcierten Krieg in Mitteleuropa. Freilich muß man die stilistisch ganz andersartigen späte- 


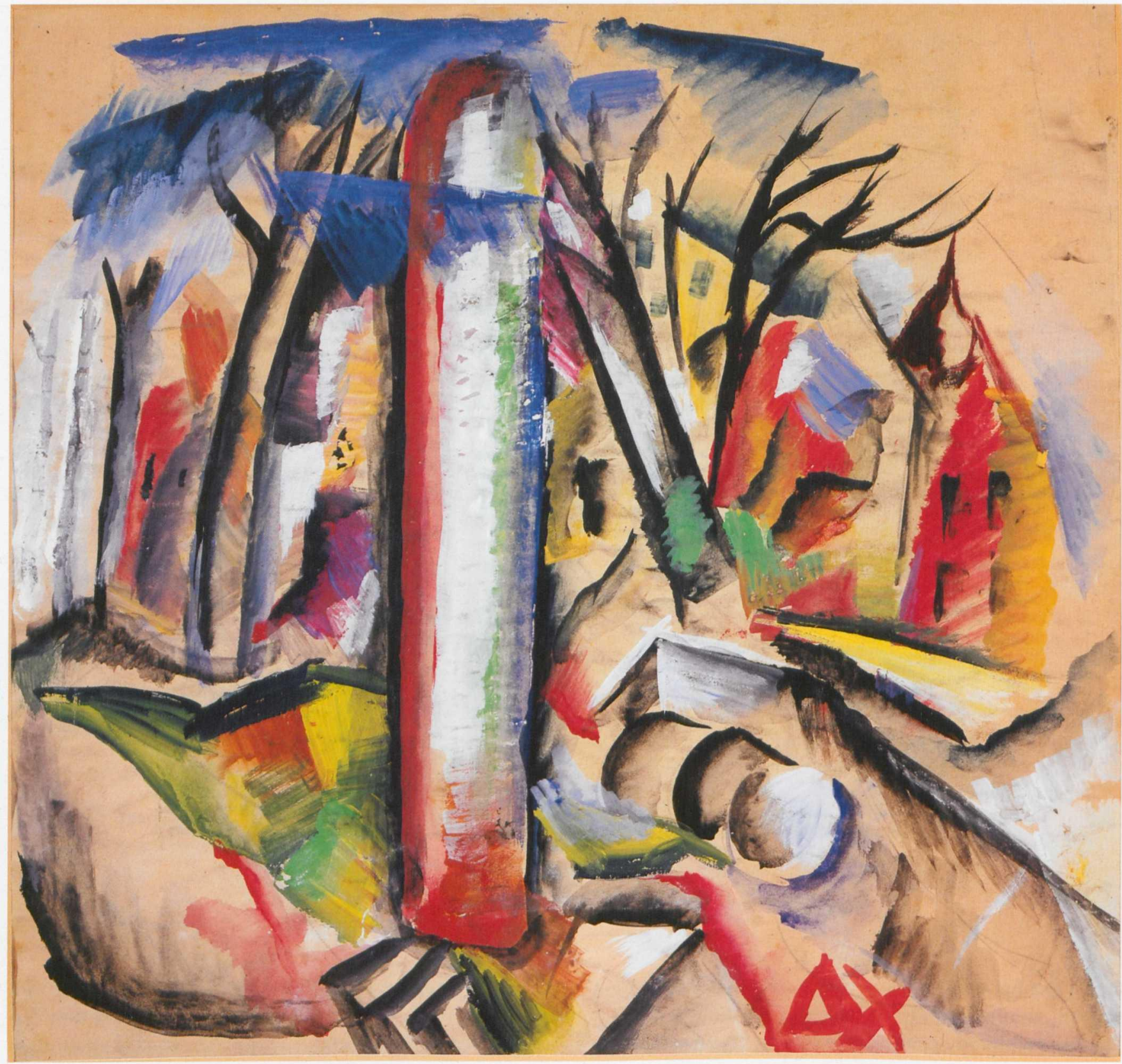

Abb. 13. Otto Dix, Landschaft mit Säule, Gouache 1917, Privatbesitz Darmstadt

ren 50 Radierungen von 1924, die Mappe "DER KRIEG«, bei dieser Gesamtdeutung mit heranziehen, um zu erkennen, daß Dix in dieser zweiten Phase seiner Kriegsverarbeitung ${ }^{36}$ statt der Simultaneität der Dynamik der Kriegsereignisse nun 1924

$36 \mathrm{Zu}$ den verschiedenen Phasen der künstlerischen Verarbeitung bzw. Gestaltung der Kriegserlebnisse durch Dix: 1. Phase 1915-1918; 2. Phase um 1922/23 mit neuen Zeichnungen aus dem Gedächtnis und vor allem dem Gemälde »Schützengraben" (auf der Wanderausstellung "Entartete Kunst 1937-39, im Januar 1940 vom Händler B. Boehmer gekauft, seither verschollen), anschließend die Radierungenmappe "DER KRIEG « 1924; die 3. Phase mit den großen Holztafeln "Krieg«, dem Triptychon in Dresden, ferner „Flandern« von 1934. (Berlin, Neue Nationalgalerie). ganz nahsichtige Details des furchtbaren Sterbens sozusagen in "Protokollen" (Kinkel) lieferte.

Die Zeichnungen von 1915-18 verkörpern das fernsichtige, dynamische Gesicht des Kriegsgeschehens; die Radierungen von 1924 aber sind das ex-

Vgl. dazu D. Schubert, Dix und der Krieg, in: Pazifismus (wie Anm. 15), 1985, S. 185-202; Uwe M. Schneede, Besprechung Conzelmann 1983, in: F.A.Z. 26. Nov. 1983; Fritz Löffler, Otto Dix und der Krieg, Leipzig 1986. - Mathias Eberle, First World War and Weimar Artists, London 1985, deutsche Ausgabe: Der Weltkrieg und die Künstler der Weimarer Republik, Stuttgart/Zürich 1989, S. 31; der wenig konkrete Beitrag von R. Beck, Krieg, in: Katalog der Ausst. Dix, (wie Anm. 2) 1985 , 11-13; D. Schubert, DIX (1980), 3. Aufl. 1991, S. 21 f., $66 \mathrm{f}$. und 96. O.K. Werckmeister: Radical Art History, in: ART JOURNAL, 42,1982 , S. 284 f. 


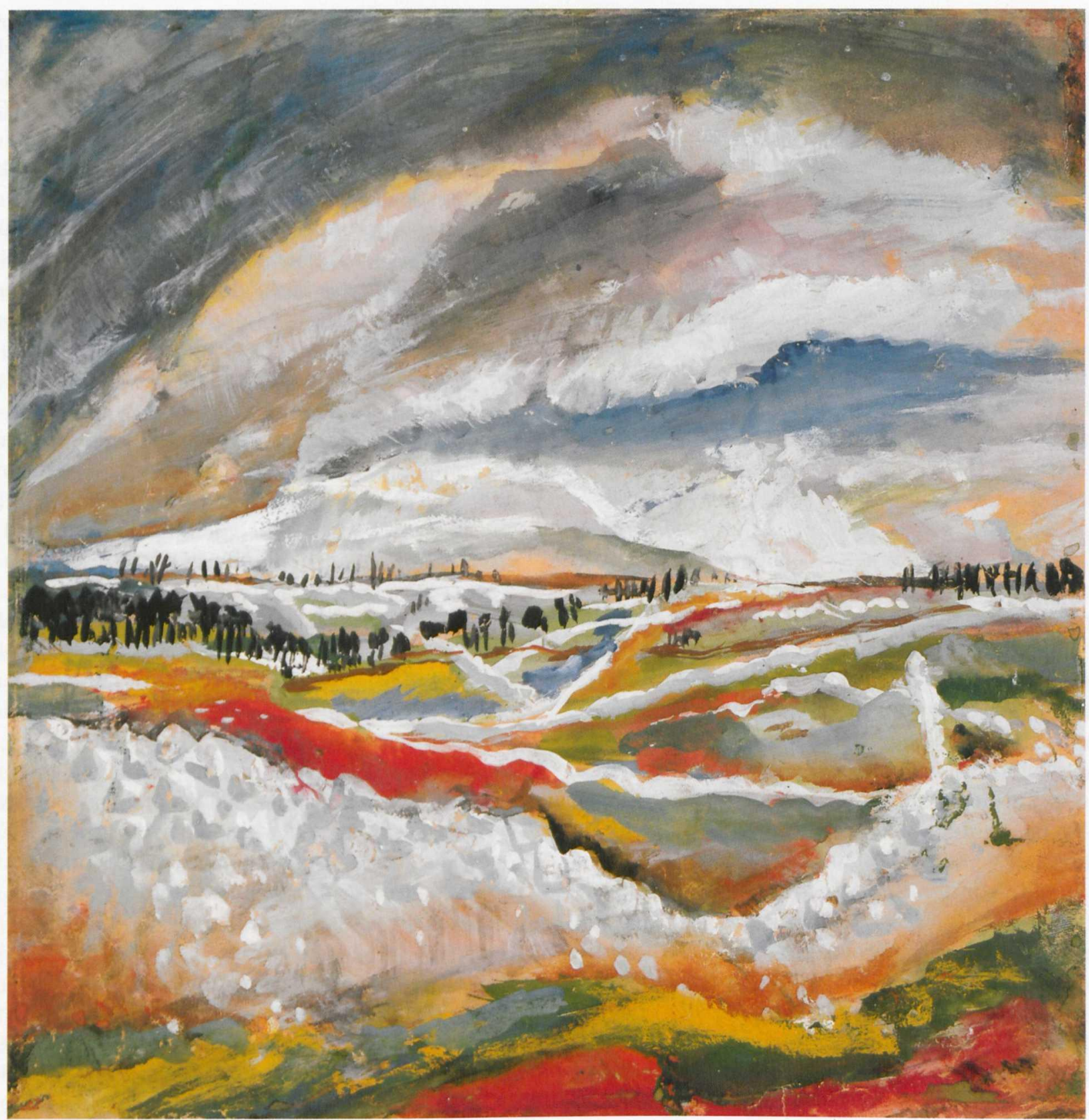

Abb. 14. Otto Dix, Gräben vor Reims I, Gouache Frühjahr 1916, Privatbesitz Mannheim

trem nahsichtige, wirkliche Antlitz des Krieges und besonders seiner Folgen: das Sterben der Menschen, - um mit der Definition des französischen Malerrealisten Courbet zu sprechen: der vollkommene Ausdruck einer existierenden Sache, »la vrai vérité«. ${ }^{37}$

Innerhalb des Spektrums der Arbeiten von Dix im Kriege verkörpert die hier publizierte Gouache kein Kampfgeschehen, keinen Granateinschlag, keinen Frontverlauf, eher eine Szenerie am Rande der Schlachtfelder in Nordfrankreich, der Somme oder Flandern. Die architektonische Säule aus ver-

37 Klaus Herding, Malerei als Widerspruch - Gustave Courbet, Frankf./M. 1976.

Vgl. hier auch Emile Zolas Unterscheidung von August 1864 gangener Zeit wird innerhalb der Natur zu einem rätselhaften Gerät, das zwischen dem Rot der Erde und dem transzendenten Blau des Himmels ungestüm vermittelt. Alles überragend, steht sie zugleich fremd gegen alles, und sie ist ohne Bewegtheit, d.h. das Unnatürliche, das Künstliche.

Die Signatur dieser Gouache zeigt die bereits bekannte Art der quasi kubistischen Deformation, d.h. D und $X$ werden als Formen aneinandergerückt; sie werden zu einer eigenständigen Form innerhalb des Formgeflechts der Bild-Gestalt. Leuchtend karminrot steht die Signatur wie die blut-

in seinem Brief an Anthony Valabrègue: "L'écran classique "l'écran romantique - „l'écran realiste« (Emile Zola, Correspondance, Paris 1908, p. 16). 
roten Teile im Hintergrund vor dem hellen Ocker des Malgrundes. Mit diesem Element, den stegartig geknickten Formen im Mittelgrund und den gestisch geformten Baumstämmen steht das Bild zusammen mit der zeitgleichen Gouache »Grabkreuze« mit ebenfalls roter Signatur (Museum Albstadt) ${ }^{38}$ zwischen der Amiens-Zeichnung (Abb. 12) und den im Spätherbst 1917 entstandenen Kreideund Kohlezeichnungen. Ich würde als Datierung also die Wochen im Herbst 1917 vorschlagen, als Dix im September-Oktober in der Herbstschlacht in Flandern eingesetzt war (It. Militärpaß bis 4.10.). Da die rückseitige Betitelung keinen Ortsnamen nennt, sondern nur das Wort »Säule«, ist eine nähere Bestimmung der Ortschaft kaum möglich.

Den zeitlichen Abstand und die Differenz in den Gestaltungsprinzipien erkennt man bei einem Vergleich mit einer Gouache aus den Kriegsmonaten in der Champagne, d.h. Winter 1915-1916 während der Stellungskämpfe bei Aubérive und vor Reims: Dix hat die Gräben vor Reims in zwei farbigen Gemälden (Gouachen) festgehalten, in einer düsteren Szenerie mit drohenden Wolkengebilden und in einer lichteren, in eisigem Blau gehaltenen Szene. Beide Gouachen sind aus der Literatur bekannt ${ }^{39}$ und verdeutlichen in ihren realistischen, runden Formen (wie der "Sprengtrichter" in Dresden) und in den kleinteiligen Weiß-Tupfern und kleinen schwarzen Pfählen und Bäumen eindeutig die Stillage vor den kubistisch-futuristischen FormSystematisierungen des Jahres 1917: „Gräben vor Reims I» (Abb. 14) gehört entgegen dem Verzeichnis bei Suse Pfäffle neben und zeitgleich zu "Stellungskrieg « (G 1916/5), "Betongraben mit Blumen" (G 1916/18), »Granattrichter» (in Bad Nenndorf, G 1916/22), »Schützengraben « (G 1917/25) und »Grab eines Franzosen/Champagne (G 1915/18). Gemeinsam mit unserer Abb. 9 zeigt diese Werkgruppe die charakteristischen Formen der Monate bis Juli 1916 in der Champagne.

Der historische Kontext solcher Behandlung

38 Das mit unserer Gouache sehr ähnliche Bild »Grabkreuze" war auch 1991 in Stuttgart/Berlin ausgestellt (vgl. Anm. 1); dazu Katalog der Ausstellung DIX, Städt. Galerie Museum Albstadt, hrsg. von Alfred Hagenlocher/Adolf Smitmans, Albstadt 1991, Nr. 44; ferner in diesem Katalog der Beitrag von Hans Höfchen, Zum Zeichner Dix, S. 9-25 mit dem berechtigten Appell, bei den Zeichnungen mehr zu differenzieren (Anm. 65).

Die jüngst bei Christie's London, Okt. 1995, lot 142 versteigerte Gouache "Souchez-Tal/Tümpel " gehört stilistisch genau in die Nachbarschaft der Souchez-Studie im Kupferstich-Kab. Dresden, die kleiner ist (vgl. Kat. Dresden, von U. Lehmann, 1991, Nr. 35 mit Vorschlag 1917). Beide zeigen in den Ästen der Bäume runde Formen, auch die Signatur ist noch nicht kubistisch wie 1917. Dix war im Souchez-Tal (in der Nähe von Angres) im Herbst 1916 in den Stellungskämpfen um Arras (Militärpaß p. 11); eine Datierung in die Monate künstlerischer und schriftlicher Dokumente von Malern und Bildhauern im 1. Weltkrieg ist natürlich ohne Frage auch das Verhältnis von Front und Heimat; die methodische Grundlage hierfür hat O.K. Werckmeister in seinem Klee-Buch geliefert (siehe Anm. 6). Für Otto Dix ist diese Differenzierung hinsichtlich sozusagen privater und der für den Kunstmarkt bestimmten Blätter und Gouachen von 19151918 noch nicht geleistet. Sie wäre wirkliches Neuland innerhalb der Dix-Forschung, ebenso wie eine kritisch kommentierte Neuausgabe seiner Kriegszeichnungen fehlt.

Ich spreche diese Desiderate hier zum Schluß nur an, um meine Sicht auf die derzeitige Lage der Dix-Literatur, die sich zwischen Pseudo-NeulandPostulaten (Rainer Beck), kritiklosem Positivismus und biografistischem Elan (öffentlicher Briefabtausch zwischen L. Tittel und R. Pfefferkorn 1992 um die intimen sexuellen Skizzen des alten Dix) bewegt, zu vermitteln. Eine komplette Ausgabe der wichtigsten Kriegszeichnungen, die noch erhalten sind, wäre demgegenüber tatsächlich ein großes, wichtiges Projekt angesichts dessen, daß Dix den 1. Weltkrieg von allen Künstlern des 20. Jahrhunderts am intensivsten wiedergab.

Wann Dix an welchen Ausstellungen im Krieg mit welchen Gouachen oder Kreidezeichnungen vertreten war, ist ein vergleichsweise kleines Feld. Aber trotzdem kann es hier in diesem Text in allen Einzelheiten nicht vorgelegt werden, weil es den Rahmen dieses Beitrages sprengen würde; nur das Wichtigste: Tatsache ist, daß Dix nicht nur an Ausstellungen in der Heimat teilnahm, er war auch sehr interessiert, Zugang zu solchen in Dresden im Kreise der Expressionisten und der Galerien Arnold und Richter zu finden. Seine Beteiligung mit 11 Zeichnungen an der »Zweite(n) Ausstellung Dresdner Künstler, die im Heeresdienste stehen «, ${ }^{40}$ vom 27. September bis 29. Oktober 1916 in der Galerie Ernst Arnold, Dresden/Schloßstraße, wirft ein erhellendes Licht auf jene Frage nach Unterschei-

August-Oktober 1916 für die beiden Souchez-Bilder (die S. Pfäffle nicht gemeinsam ansetzte) wäre m.E. also angebracht. 39 O. Conzelmann, 1983 (wie Anm. 10), Abb. 191; Katalog Dix, hrsg. v. R. Beck, 1985, Nr. 24 (Farbe); der "Granattrichter in Stern- bzw. Blütenform war in der großen Retrospektive Stuttgart/Berlin 1991, Kat. S. 63. - Suse Pfäffle (wie Anm. 1) 1991, S. 266 schreibt zu diesem blütenförmigen Trichter mit weißen Tupfern zwar, „die stilistische Ähnlichkeit mit G 1915/8 spricht dafür, daß das Blatt um 1916 entstanden ist«, ordnet beide aber nicht direkt einander zu und datiert das eine 1915 , das andere 1916.

Die Signatur von G 1915/8 ist im Duktus der Jahre 1960/62, d.h. später hinzugefügt worden, offenbar während der Auswahl der Blätter für die von Jean Cassou in St. Gallen besorgte 1. Ausstellung der Kriegsarbeiten (Galerie Erker, Katalog "DER KRIEG«, St. Gallen 1961-62, Text von Jean Cassou). 40 Die Kenntnis dieser Ausstellungsbeteiligung von Dix geht 
dung in Kunst für sich und Kunst für die Heimat, um mit Fr.Nietzsche zu sprechen: "monologische Kunst» und »Kunst für Zeugen « (Fröhliche Wissenschaft).

Hier müssen nicht nochmals die elf Titel aufgelistet werden, da sie inzwischen im Dresdner Katalog der Dix-Zeichnungen 1991 publiziert sind (vgl. Anm. 11). Die Identifizierung der Blätter ist z.Zt. nicht exakt zu machen: Nr. 241 »Schlafender Soldat, Pont Faverger « dürfte das Blatt sein, das Löffler in seiner Monographie abbildete und das das Datum 2.11.1915 trägt (heute in Dresden) $;^{41}$ das Blatt "Straße Souplet-Aubérive« Nr. 240 wird durch die Geraer Feldpostkarte 29 reflektiert; eine Zeichnung mit der Konnotation "Dontrien« wie Nr. 243 kenne ich bislang nicht; freilich gibt es den Titel der erwähnten Radierung »Trichterfeld bei Dontrien«. Die Nr. 238c »Stollen zum Unterstand « könnte das heute im Museum Friedrichshafen aufbewahrte Blatt von $29 \times 28 \mathrm{~cm}$ sein; es ist auf April $1916 \mathrm{zu}$ datieren. ${ }^{42}$ Laufgräben mit Drahtverhauen, Schützengräben, zerstörte Dörfer und Granattrichter kommen besonders in den Zeichnungen der Wochen um Aubérive vor (Abb. 3), also im Frühjahr 1916. Eine mögliche Identität schlage ich noch vor für die Ausst.Nr. 239 »Minenstollen«, nämlich das Stuttgarter Blatt, das den Ortsnamen "Aubérive « und das Datum 16 trägt (Abb. 15). ${ }^{43}$

Offenbar hat Dix lediglich die elf Zeichnungen ausgestellt und keine Gouachen. Mir scheint aber, daß gerade die besten der Gouachen von 1916 und 1917 - mehr als die teils spontanen Kreidezeichnungen - für die erhofften Erfolge in Heimat-Ausstellungen besonders bestimmt waren. Denn sie zeigen stärker als die Schwarzweißarbeiten, in welchem Maße Dix "modern" sein konnte, d.h. die Formmodi kubistischer, expressionistischer und futuristischer Gestaltung frei adaptiert hatte. Und die glühenden Farben der Gouachen vermögen den erhöhten Reiz einer Kunst für Publikum zu tragen, zumal in ihnen Tatsachen wie Volltreffer eines Soldaten oder Sterbende im Granatfeuer nicht auftauchen.

Die Frage, die also zu behandeln wäre, ist die nach den Dix-Ausstellungsbeteiligungen in der Hei-

auf den Hinweis von O.K. Werckmeister im Herbst 1987 zurück (vgl. Anm. 12); siehe auch Hans U. Lehmann, in: DixKat. Dresden (wie Anm. 11), S. 54.

41 Fritz Löffler, DIX - Leben und Werk, 4. Aufl. Dresden 1977, S. 10; Hans U. Lehmann (wie Anm. 40), Nr. 18.

42 Vgl. Lutz Tittel (Hrsg.), Katalog der Dix-Sammlung (wie Anm. 29), Friedrichshafen 1992, Nr. 17 (falsch 1915 datiert). Die Nr. 243 »Dontrien« könnte natürlich - wenn die rückseitige Numerierung verläßlich ist - das zertrümmerte Dorf in Dresden (Lehmann 1991, Nr. 27) sein; dann wäre nicht der

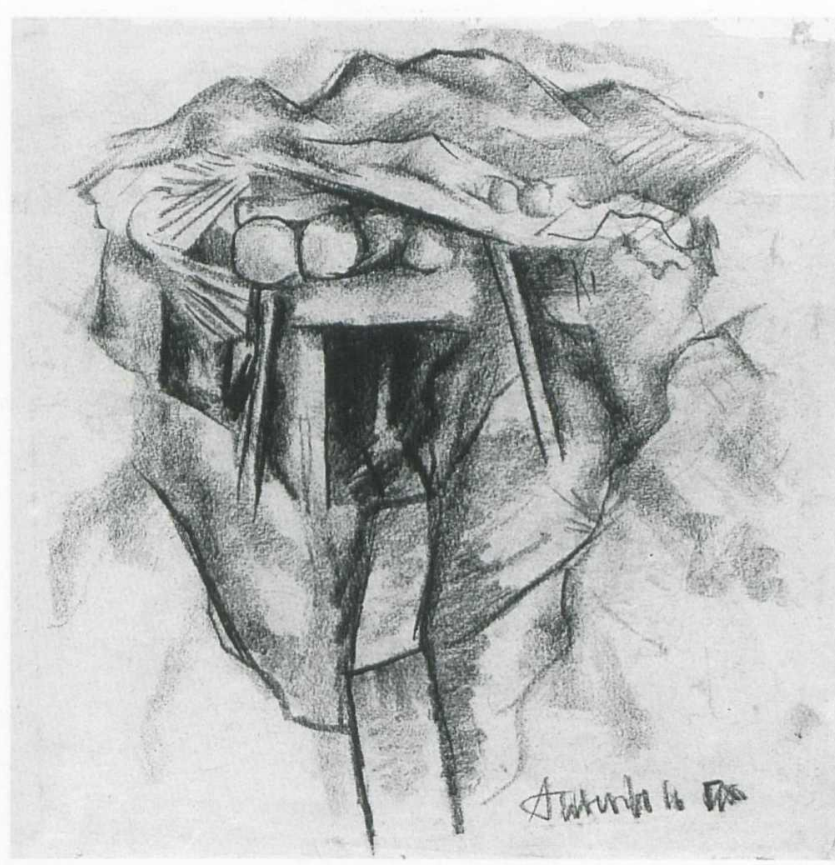

Abb. 15. Otto Dix, Minenstollen bei Aubérive, Frühjahr 1916, Kreide, Galerie der Stadt Stuttgart

mat mit Kriegsbildern zwischen 1915 und 1918. Über die in Dresden 1916 hinaus wissen wir bislang nur noch von zwei Ausstellungen. Auf der Fotokarte "Dix auf Urlaub« von Mitte August 1917 aus Brügge bittet Dix seine Freundin H. Jakob:

"Am 15. Aug. wird die Ausstellung in der Lennéstr. eröffnet, woselbst ich auch Zeichnungen ausgestellt habe. Würden Sie mir die Zeitungen, welche Kritiken über diese Ausstellung bringen, zusenden? Ich danke Thnen im voraus bestens dafür. ${ }^{44}$ In dieser Straße war der Sitz der KünstlerVereinigung Dresden; in deren Herbstausstellung 1917 wurde von Dix jedoch lediglich als Nr. 110 eine »Geißelung« gezeigt und nicht mehrere Blätter, die Dix ohne Zweifel einreichte. Das erstaunt; wurden andere Kriegsblätter etwa refusiert?

Eine dritte Ausstellung vor Kriegsende kam wohl nicht zustande. Dix schrieb am 4. Januar 1918 aus dem Felde an den Bürgermeister von Gera:

»Euer Hochwohlgeboren!

Ich gestatte mir hierdurch die höfliche Anfrage, ob es mir möglich ist, Ende Februar - Anfang März d.J. in den Räumen des Städt. Museums zu Gera ei-

Ort Aubérive, sondern eben Dontrien von Dix gezeichnet, ein Schluß, den Lehmann nicht zog.

43 Bestandskatalog Galerie der Stadt Stuttgart (wie Anm. 8), von Brigitte Reinhardt, 1989, Nr. 66.

44. U. Rüdiger, Grüsse (wie Anm. 4), S. 100; "Lauenstraße« ist ein Lesefehler: es handelt sich um die Lennéstraße, Sitz der mehr liberalen Künstler-Vereinigung Dresden, die auch Expressionisten zeigte, im Gegensatz zur reaktionären »Kunstgenossenschaft«. Diese Ausstellungsbeteiligung von Dix 1917 in der Heimat auch fehlerhaft bei A. Strobl: Malerkarriere (Anm. 11), 1996, S. 242. 


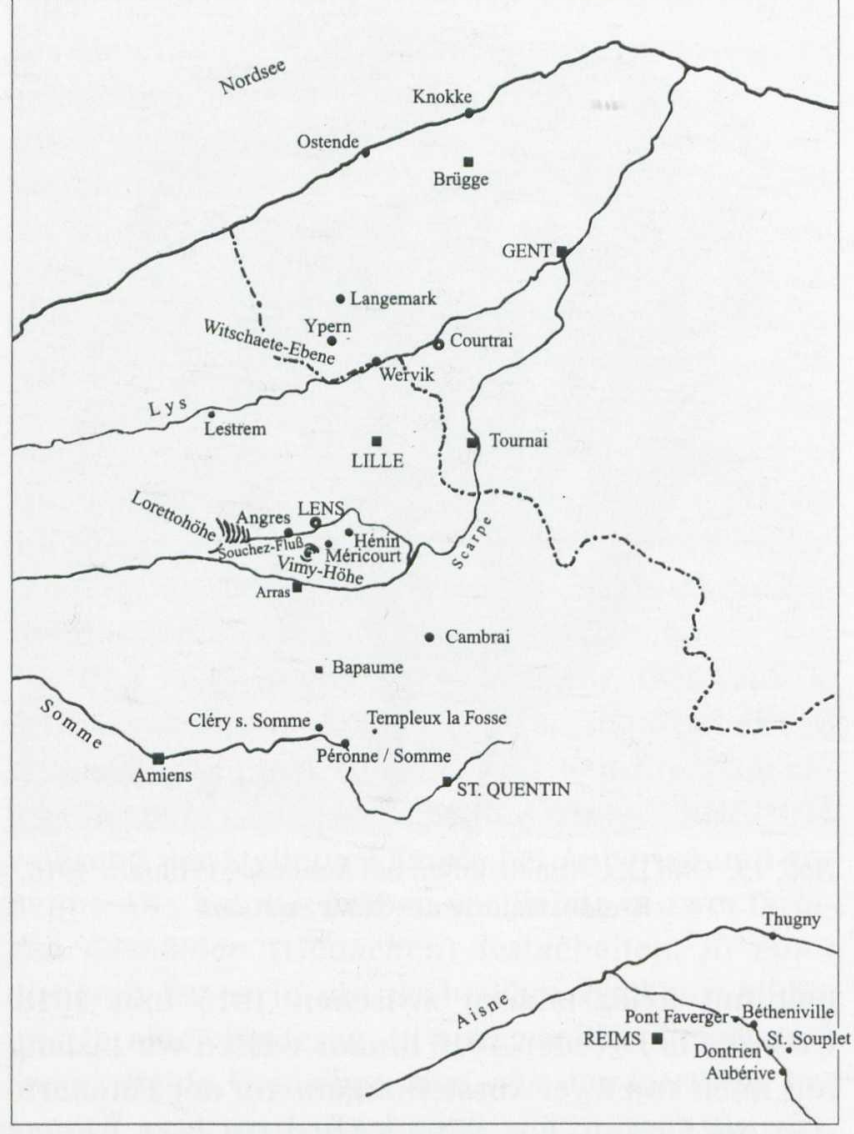

Übersichtskarte zu den Einsätzen von Unteroffizier Dix an der Westfront 1915-1918

ne Kollektion Kriegsbilder und Zeichnungen, 10-15 Stück, auszustellen.

Ich bin geborener Untermhäuser ... meine Arbeiten sind von expressionistischer Art und diese jüngste Richtung ist vielleicht für die Geraer Kunstkennerschaft neu und interessant. Ich hoffe bald auf günstige Antwort.

Hochachtungsvoll OTTO DIX Ut.ffz.DIX I. MGkomp., R.I.Reg.102« ${ }^{45}$

Was solche Selbstreklame belegt, ist ein Selbstbewußtsein von besonderer Art bei Dix. Er rechnet sich nicht zu den sog. „Futuristen«, sondern er definiert seine Arbeiten als expressionistische, d.h. ihm kam es auf den Gehalt und die Form an. Und was drittens für unseren Zusammenhang wichtig scheint: Dix unterscheidet nun klar zwischen "Kriegsbildern« (Gouachen) und den Zeichnungen (Kreide, Kohle), und er will eine Mischung von beiden zeigen.

45 Dieser Brief befindet sich im Dix-Haus, Gera-Untermhaus. 46 Hans Kinkel, Protokolle der Hölle, 1968, Nr. 42; Katalog Auktion Lempertz 561, Köln 1977, Nr. 187, Kreide $26 \times 29 \mathrm{~cm}$ (Privatbesitz); D. Schubert, in: KULTUR und KRIEG, hrsg. von W.J. Mommsen, 1995, Abb. 13, S. 191

4.7 Vgl. dazu Dieter Gleisberg, Conrad Felixmüller und die Gründung der Dresdner Secession Gruppe 1919, in: Dezennium - Verlag der Kunst Dresden 2, Dresden 1972, S. 162 f. Dieter Gleisberg: Conrad Felixmüller - Leben und Werk,
Die von O.K. Werckmeister für Paul Klee so fruchtbar entwickelten Fragen können für Dix erst einer weitgehenden Beantwortung entgegensehen, wenn wir alle Ausstellungsbeteiligungen von ihm 1915/18 - ab 1919 stellt er mit der »Gruppe 1919« in Dresden aus - und die Identität der Blätter zu klären vermögen. Erst nach solcher Rekonstruktion könnte man das Verhältnis von Erfolg oder Mißerfolg der Kriegsthematik durch Dix in der Heimat beurteilen. Freilich, Dix hat sich kaum nach dem herrschenden Geschmack richten können oder brauchen, da er keinen Vertrag mit einer Galerie hatte. Jünger als Paul Klee, mußte er sich erst durchsetzen. Überblickt man seine Kriegszeichnungen und Gouachen, so haben wir eine enorme Fülle an Blättern voll Wucht, Intensität und geballter Kraft, ausgehend von einem enormen Lebens- und Überlebens-Willen, Arbeiten, die einen völlig sicheren Zeichner dokumentieren, der zu abstrahieren vermag, um das Wesentliche in einer Verdichtung zu zeigen. Auch ist sich Dix nicht nur in der Darstellung des Erlebten und Gesehenen treu geblieben. Er zeichnete dazwischen auch Träume, Visionen, Wünsche und Sehnsüchte, wie z.B. die Friedens-»Finale«-Zeichnung in Dresden (1917), und Themen die mit der männlichen Sehnsucht nach Leben, Lust, Schönheit und Sexualität im Sinne des Vitalismus ihres Autors zu tun haben: 1917 datiert ist das Blatt (Privatbesitz) mit einem kubistischen Frauenakt, das Weib als Partnerin und Lustobjekt des Mannes und als Gebärerin, Titel »Fruchtschale $;^{46}$ eventuell in den Winter 1917 auf 1918 zu setzen (Rußland?).

Wie sehr Dix von dieser Thematik bewegt und getrieben war, belegt der Brief nach dem Kriege, von Februar 1919, an den Freund Kurt Günther in Gera: "Ich habe zwei größere Gemälde angefangen ... Leda und Weib mit Fruchtschale. Die Ideen trage ich schon seit 5 Jahren ... Wir, die radikalen Dresdner haben eine Sezession gegründet ... Wir erhalten Ausstellungsräume bei Richter .... ${ }^{47}$

Fotonachweis

Foto Kabus, Dix-Stiftung Vaduz: 1, 5; Kunsthaus Lempertz Köln: 2; Staatliche Museen zu Berlin, Kupferstichkabinett: 3, 11; Galerie der Stadt Stuttgart: 4, 15; Gera Museum/Foto Schenke: 6; Villa Grisebach Berlin/Foto Bartsch: 7; D. Schubert, Heidelberg: 8, 9, 10, 12, 14; Renate Deckers-Matzko, Heidelberg: 15

Dresden 1982, S. 44-45. - Katalog »Kunst im Aufbruch Dresden 1918-1933 «, Kunstsammlungen Dresden 1980, S, 41 (Text von Fritz Löffler). - Ruth Gleisberg: Conrad Felixmüller- Werke und Dokumente, Staatl. Lindenau-Museum Altenburg (Expositionen 4), Altenburg 1982. - D. Schubert, DIX, 1991, S. 36 - Ferner Rainer Beck, Otto Dix, Konstanz 1993, S, 63. - Lothar Fischer, Otto Dix - ein Malerleben in Deutschland, Berlin 1981, S. 26 